\title{
Hybrid State-Space Fuzzy Model-Based Controller with Dual-Rate Sampling for Digital Control of Chaotic Systems
}

\author{
Young-Hoon Joo, Leang-San Shieh, Senior Member, IEEE, and Guanrong Chen, Fellow, IEEE
}

\begin{abstract}
In this paper, we develop a hybrid state-space fuzzy model-based controller with dual-rate sampling for digital control of chaotic systems. Takagi-Sugeno (TS) fuzzy model is used to model the chaotic dynamic system and the extended paralleldistributed compensation technique is proposed and formulated for designing the fuzzy model-based controller under stability conditions. The optimal regional-pole assignment technique is also adopted in the design of the local feedback controllers for the multiple TS linear state-space models. The proposed design procedure is as follows: an equivalent fast-rate discretetime state-space model of the continuous-time system is first constructed by using fuzzy inference systems. To obtain the continuous-time optimal state-feedback gains, the constructed discrete-time fuzzy system is then converted into a continuoustime system. The developed optimal continuous-time control law is finally converted into an equivalent slow-rate digital control law using the proposed intelligent digital redesign method. The main contribution of this paper is the development of a systematic and effective framework for fuzzy model-based controller design with dual-rate sampling for digital control of complex such as chaotic systems. The effectiveness and the feasibility of the proposed controller design method is demonstrated through numerical simulations on the chaotic Chua circuit.
\end{abstract}

Index Terms - Chaotic Chua's circuit, digital redesign, dualrate sampling, fuzzy control, optimal control, pole placement.

\section{INTRODUCTION}

$\mathbf{R}$ ECENTLY, increasing attention has been focused on developing techniques for the control of chaotic dynamical systems [1]. Chaos is a main characteristic of complex dynamical systems. It is usually difficult to predict a longterm future behavior of a chaotic system [1]. Because of its unpredictability and irregularity, chaos could lead systems to undesirable performance-degraded situations. Therefore, in many cases, chaos should be avoided or purposely controlled [16].

On the other hand, we have also witnessed rapidly growing interest in making control systems more intelligent. Among various artificial intelligent approaches, fuzzy control has enjoyed remarkable success in many applications [2]. Moreover, recent advances in fuzzy control have laid a solid foundation

Manuscript received October 26, 1998. This work was supported in part by the U.S. Army Research Office under Grant DAAG-55-98-1-0198 and by the KOSEF (Korean Science and Engineering Foundation).

Y.-H. Joo is with the Department of Control and Instrumentation Engineering, Kunsan National University, Kunsan, Chonbuk, 573-701 Korea.

L.-S. Shieh and G. Chen are with the Department of Electrical and Computer Engineering, University of Houston, Houston, TX 77204 USA.

Publisher Item Identifier S 1063-6706(99)06732-6. for intelligent control of different types of nonlinear processes, including chaotic systems. For example, Chen [1], [17] applied fuzzy inference systems to identify and control chaotic systems with success. Wang et al. [2], [18] introduced fuzzy modeling for chaotic systems and used a parallel-distributed compensation (PDC) for control. Lately, they addressed the issues of regulation as well as model-following chaos control [12]. In this paper, we continue these efforts to develop a new hybrid state-space fuzzy model-based control technique for digital control of continuous-time chaotic systems.

Most real dynamic systems are described by continuoustime models. The rapid advances in digital control theory and the availability of high-performance low-cost microprocessors have demanded the development of digital controllers for analog systems. Since the design of a continuous-time system using a digital controller is usually not closely matched with the continuous counterpart in a real environment, it is desirable to develop a hybrid control scheme [3].

In the past, adaptive control concepts [3]-[7], [10] have been extended to combine the continuous-time control theory with a discrete-time adaptive mechanism (for updating the controller parameters) for self-tuning control of hybrid systems. In particular, Karwick [5] developed a state-space self-tuning method for pole assignments of continuous-time systems and Helliot [6] proposed a discrete adaptation technique for the control of continuous-time processes.

For practical realizations of such well-developed, advanced control algorithms for highly nonlinear systems, it becomes necessary to utilize dual-rate sampling schemes. A fast-rate sampling scheme is used to perform parameter identification and fast-rate controller design, while a slow-rate sampling scheme is employed to establish a slow-rate controller that takes into account the performance of the analog controller and the computational delays in the identification and control processes. It has long been experienced that the conventional digital redesign schemes can hardly control complex continuous-time nonlinear systems such as chaotic systems consisting of multiple state-space systems.

In order to resolve this problem, in this paper, we present a hybrid state-space fuzzy model-based controller with dualrate sampling for digital control of complex such as chaotic systems. Takagi-Sugeno (TS) fuzzy model is first used to model the chaotic dynamic system and then the extended parallel-distributed compensation (EPDC) technique is proposed and formulated for the design of a fuzzy model- 
based digital controller. The optimal regional-pole assignment technique is also adopted (together with stability conditions) for the design of multiple local linear state-space models. Using these techniques, once the fuzzy model representation of a dynamic system is obtained, we can further develop some new fuzzy controller design methods to the digital control of the complex system. More precisely, an equivalent fast-rate discrete-time state-space model of the continuoustime system is first constructed by using the identified fuzzy inference systems. To obtain the continuous-time optimal statefeedback gains under slow-rate sampling, the constructed fast-rate discrete-time fuzzy system is then converted into a continuous-time fuzzy system with slow-rate sampling. The developed optimal continuous-time control law is finally converted into an equivalent slow-rate digital control law using the proposed intelligent digital redesign method to find the optimal controller. The basic idea here is to design stable local feedback controllers based on local dynamic models using the optimal pole-assignment technique and then construct a stable global controller from these multiple optimal local controllers. The stability of the resulting controller is guaranteed by some new stability conditions developed based on the rigorous Lyapunov stability theory. This overall proposed design procedure presents a systematic and effective framework for integration of modeling and digital control of complex dynamic systems such as chaotic systems.

This paper is organized as follows. Section II describes the TS fuzzy model with PDC as a controller and the fuzzy modeling of the chaotic Chua circuit, which is used as an illustrative example. The digital redesign method in hybrid statespace fuzzy model-based controller is developed in Section III. In Section IV, hybrid state-space fuzzy model-based control scheme is described with stability conditions. In Section V, the chaotic Chua circuit modeled in Section II is used as a testbed for evaluation and demonstration of the new control method. Finally, conclusions are drawn in Section VI with some discussions.

\section{FuZZy System Modeling}

This section first reviews two representative fuzzy model structures, namely the TS fuzzy model proposed in [11], [29] and its closed-loop version with a controller as well as the state-space partition model studied in [24]-[28]. The main characteristic of a TS fuzzy model is to express the local dynamics of each fuzzy rule by a linear system model and the overall fuzzy model of the system is obtained by fuzzy blending of all the local linear system models [2]. To be specific, we will discuss the fuzzy modeling of the chaotic Chua circuit in this section, which will also be used as an example for the controller design later in the paper.

\section{A. Two Representative Fuzzy Model Structures}

Consider a nonlinear dynamic system in the canonical form

$$
\dot{\boldsymbol{x}}=\boldsymbol{f}(\boldsymbol{x})+\boldsymbol{g}(\boldsymbol{x}) \boldsymbol{u}(t)
$$

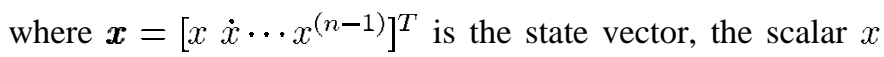
is the output state variable of interest, and $\boldsymbol{u}$ is the system control input. In (1), $\boldsymbol{f}(\boldsymbol{x})$ is a known nonlinear continuous function of $\boldsymbol{x}$ and the control gain $\boldsymbol{g}(\boldsymbol{x})$ is a known nonlinear continuous invertible function of $\boldsymbol{x}$. This nonlinear system can be approximated by the TS fuzzy model, as discussed in [11], [29].

A TS fuzzy system is described by a set of fuzzy IF-THEN rules, where each rule locally represents a linear input-output realization of the system over a certain region of the statespace. The overall system is then an aggregation of all such local linear models. More specifically, a TS fuzzy system in the continuous-time case is formulated in the following form:

$$
\begin{gathered}
\text { Rule } i: \quad \text { IF } x_{1}(t) \text { is } M_{1}^{i} \text { and } \cdots \text { and } x_{n}(t) \text { is } M_{n}^{i} \\
\text { THEN } \dot{\boldsymbol{x}}_{c}(t)=A_{i} \boldsymbol{x}_{c}(t)+B_{i} \boldsymbol{u}_{c}(t) \\
(i=1,2, \cdots, q)
\end{gathered}
$$

while the consequent part in the discrete-time case is represented by $\boldsymbol{x}_{d}(t+1)=F_{i} \boldsymbol{x}_{d}(t)+G_{i} \boldsymbol{u}_{d}(t)$ in (2).

Here, $M_{j}^{i}(j=1, \cdots, n)$ is the fuzzy set, $q$ is the number of rules, $\boldsymbol{x}(t) \in \Re^{n}$ is the state vector, $\boldsymbol{u}(t) \in \Re^{m}$ is the input vector, $A_{i} \in \Re^{n \times n}$ and $B_{i} \in \Re^{n \times m}, x_{1}(t), \cdots, x_{n}(t)$ are the premise variables (which are the system states) and $\left(A_{i}, B_{i}\right)$ in the continuous-time case and $\left(F_{i}, G_{i}\right)$ in discretetime case denote the $i$ th local model of the fuzzy system, respectively. Subscripts ' $c$ ' and ' $d$ ' represent continuous-time and discrete-time case, respectively. Using the center of gravity defuzzification, product inference, and single fuzzifier, the final output of the overall fuzzy system is given by

$$
\dot{\boldsymbol{x}}_{\boldsymbol{c}}(t)=A \boldsymbol{x}_{c}(t)+B \boldsymbol{u}_{c}(t)
$$

where

$$
\begin{aligned}
A & =\sum_{i=1}^{q} \mu_{i}(x) A_{i}, \quad B=\sum_{i=1}^{q} \mu_{i}(x) B_{i} \\
\mu_{i}(x) & =\frac{\omega_{i}(x)}{\sum_{i=1}^{q} \omega_{i}(x)} \geq 0, \quad \sum_{i=1}^{q} \mu_{i}(x)=1, \quad \omega_{i}(x)=\prod_{j=1}^{q} M_{j}^{i} .
\end{aligned}
$$

Note that system (2) can be viewed as a piecewise linearization of the original nonlinear system (1) about some nominal operating points.

The zero-input system of (3) in the continuous-time case is

$$
\dot{\boldsymbol{x}}_{c}(t)=\frac{\sum_{i=1}^{q} \omega_{i}(t) A_{i} \boldsymbol{x}_{c}(t)}{\sum_{i=1}^{q} \omega_{i}(t)} .
$$

The defuzzification procedure is used to smoothly connect the neighboring local models together, so as to build up a global fuzzy model of the underlying system. To avoid a complicated defuzzification process, the premise variables are assumed to be independent of the input vector $\boldsymbol{u}_{c}(t)$. Since at any instant, the nonlinear system is represented by a linear model [described by (3)], it is clear that linear feedback control methods can be used to design a locally linear controller for the system. 
In [23]-[25], Tanaka et al. apply the PDC procedure to design a fuzzy logic controller (FLC) for the above-described TS fuzzy model, where each control rule is individually designed from the corresponding set of rules of the fuzzy model. The designed controller shares the same premise parts with the fuzzy model and has linear state-feedback laws in the consequent parts. The PDC fuzzy controller in the continuoustime model has the following rule structure:

$$
\begin{aligned}
& \text { Rule } i: \quad \text { IF } x_{1}(t) \text { is } M_{1}^{i} \text { and } \cdots \text { and } x_{n}(t) \text { is } M_{n}^{i} \\
& \text { THEN } \boldsymbol{u}_{c}(t)=-K_{c}^{i} \boldsymbol{x}_{c}(t) \\
& (i=1,2, \cdots, q)
\end{aligned}
$$

where $K_{c}^{i}$ is the feedback gain in the $i$ th subspace. The final fuzzy controller is then represented by

$$
\boldsymbol{u}_{c}(t)=-\sum_{i=1}^{q} \mu_{i}(x) K_{c}^{i} \boldsymbol{x}_{c}(t)
$$

Assume that the number of rules that fire at all times is less than or equal to $p, 1<p \leq q$. The control gain $K_{c}^{i}$ can be determined by modern linear system theory and is explained in detail in the subsequent sections. The final closed-loop system is

$$
\dot{\boldsymbol{x}}=\sum_{i=1}^{q} \sum_{j=1}^{q} \mu_{i}(\boldsymbol{x}) \mu_{j}(\boldsymbol{x})\left(A_{i}-B_{i} K_{c}^{j}\right) \boldsymbol{x}_{c}(t)
$$

As shown in (5), the consequent part of a PDC fuzzy controller represents the control law for zero-reference input. In general, inputs are represented by $\boldsymbol{u}_{c}(t)=-K_{c}^{i} \boldsymbol{x}_{c}(t)+E_{c}^{i} \boldsymbol{r}(t)$, where $E_{c}^{i}$ is the feedforward gain matrix in the $i$ th subspace and $\boldsymbol{r}(t)$ is a constant or time-varying reference input. In order to better handle this nonzero reference tracking control problem, we propose an EPDC technique in this paper, which will be further discussed later.

\section{B. Fuzzy Modeling of Chua's Circuit}

The chaotic Chua circuit, as shown in Fig. 1, is a simple electronic system that consists of one inductor $(L)$, two capacitors $\left(C_{1}, C_{2}\right)$, one linear resistor $(R)$, and one piecewise-linear resistor $(g)$. Chua's circuit has been shown to possess very rich nonlinear dynamics such as bifurcations and chaos [15]. Because of its simplicity and universality, Chua's circuit has attracted much attention and has become a prototype for the investigation of chaos [1].

The dynamic equations of Chua's circuit is described by

$$
\begin{aligned}
\dot{v}_{C 1} & =\frac{1}{C_{1}}\left(\frac{1}{R}\left(v_{C 2}-v_{C 1}\right)-g\left(v_{C 1}\right)\right) \\
\dot{v}_{C 2} & =\frac{1}{C_{2}}\left(\frac{1}{R}\left(v_{C 1}-v_{C 2}\right)+i_{L}\right) \\
\dot{i}_{L} & =\frac{1}{L}\left(-v_{C 1}-R_{0} i_{L}\right)
\end{aligned}
$$

where the voltages and current $v_{c 1}, v_{c 2}$, and $i_{L}$ are the state variables, $R_{0}$ is a constant, $g$ denotes the nonlinear resistor, which is a function of the voltage across the two terminals of $C_{1}$.

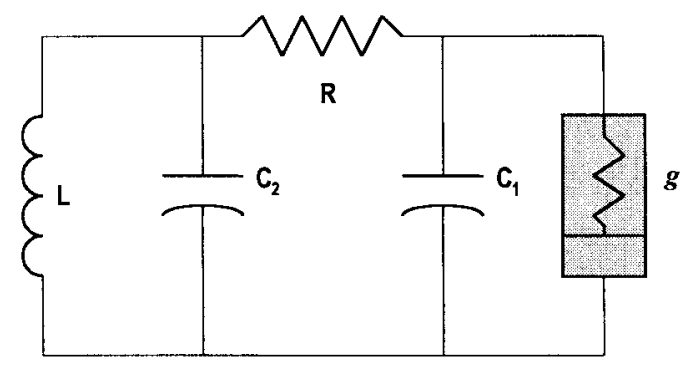

Fig. 1. Diagram of Chua's circuit.

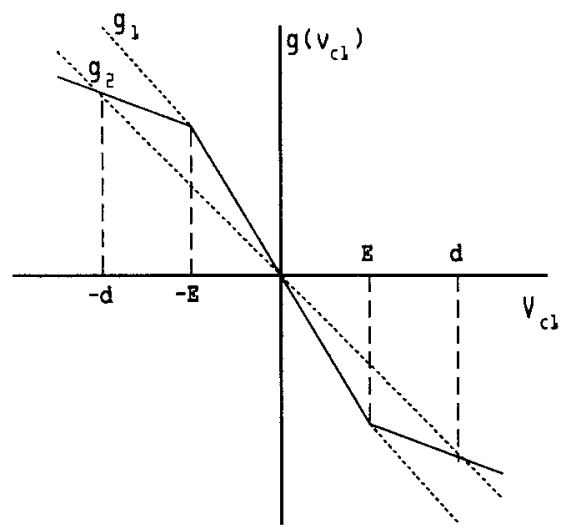

Fig. 2. Resistor characteristic in the case of a piecewise-linear function.

We consider two types of characteristics of the nonlinear resistor $g\left(v_{c 1}\right)$ : one is the original piecewise-linear function and the other is a cubic function. We only provide the resulting fuzzy models of the circuit here (for more details, see [18]).

2) Case 1: $g\left(v_{c 1}\right)$ is a piecewise-linear function

$g\left(v_{C 1}\right)=m_{b} v_{C 1}+\frac{1}{2}\left(m_{a}-m_{b}\right)\left(\left|v_{C 1}+E\right|-\left|v_{C 1}-E\right|\right)$

where $E$ is a constant voltage and $m_{a}$ and $m_{b}$ are negative, as shown in Fig. 2, which can also be represented by the three-segment piecewise-linear function

$$
g\left(v_{C 1}\right)= \begin{cases}m_{b} v_{C 1}+\left(m_{a}-m_{b}\right) E & v_{C 1} \geq E \\ m_{a} v_{C 1} & -E<v_{C 1}<E \\ m_{b} v_{C 1}-\left(m_{a}-m_{b}\right) E & v_{C 1} \leq-E .\end{cases}
$$

We want to obtain a fuzzy model in the open-loop form (4) for Chua's circuit with characteristic (9). Assuming $v_{C 1} \in$ $[-d, d], d>E>0$, the following bounds for $g\left(v_{c 1}\right)$ are obtained:

$$
\begin{aligned}
& g_{1}\left(v_{C 1}\right)=m_{a} v_{C 1} \\
& g_{2}\left(v_{C 1}\right)=\left(m_{a}+\frac{\left(m_{a}-m_{b}\right) E}{d}\right) v_{C 1}=m_{t} v_{C 1}
\end{aligned}
$$

where $m_{t} \equiv m_{a}+\left(\left(m_{a}-m_{b}\right) E / d\right)$. Chua's circuit becomes a linear system if $m_{a}$ and $m_{b}$ are the same. When $m_{a} \neq m_{b}$, the trapezoidal membership functions shown in Fig. 3 are used to model the circuit.

Denote $\boldsymbol{x}=\left[v_{C 1}, v_{C 2}, i_{L}\right]^{T}$. In order to build the intended fuzzy model, the parameter $d$ must be chosen properly. Chua's circuit with piecewise-linear characteristic for $v_{C 1} \in[-d, d]$ 


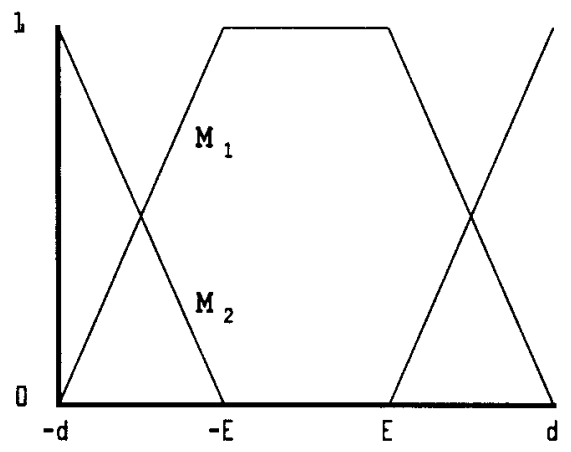

Fig. 3. Membership functions in the case of piecewise-linear $g\left(v_{c 1}\right)$.

can be exactly represented by the following fuzzy rules:

$\begin{aligned} \text { Rule 1: } & \left.\text { IF } v_{C 1} \text { is } M_{1}\left(v_{C 1}\right) \text { (near } 0\right) \\ & \text { THEN } \dot{\boldsymbol{x}}(t)=A_{1} \boldsymbol{x}(t)\end{aligned}$

Rule 2: IF $v_{C 1}$ is $M_{2}\left(v_{C 1}\right)($ near $\pm d)$ THEN $\dot{\boldsymbol{x}}(t)=A_{2} \boldsymbol{x}(t)$

where

$$
A_{1}=\left[\begin{array}{ccc}
-\frac{1}{C_{1} R}-\frac{m_{a}}{C_{1}} & \frac{1}{C_{1} R} & 0 \\
\frac{1}{C_{2} R} & -\frac{1}{C_{2} R} & \frac{1}{C_{2}} \\
0 & -\frac{1}{L} & -\frac{R_{0}}{L}
\end{array}\right]
$$

and

$$
A_{2}=\left[\begin{array}{ccc}
-\frac{1}{C_{1} R}-\frac{m_{t}}{C_{1}} & \frac{1}{C_{1} R} & 0 \\
\frac{1}{C_{2} R} & -\frac{1}{C_{2} R} & \frac{1}{C_{2}} \\
0 & -\frac{1}{L} & -\frac{R_{0}}{L}
\end{array}\right] .
$$

2) Case 2: $g\left(v_{c 1}\right)$ is a cubic function (as shown in Fig. 4)

$$
g\left(v_{C 1}\right)=a v_{C 1}+c v_{C 1}^{3} \quad(a<0, c>0) .
$$

Similarly to Case 1 , assuming $v_{C 1} \in[-d, d], d>E>0$, the following bounds for $g\left(v_{c 1}\right)$ are obtained:

$$
\begin{aligned}
& g_{1}\left(v_{C 1}\right)=a v_{C 1} \\
& g_{2}\left(v_{C 1}\right)=\left(a+c d^{2}\right) v_{C 1}=m_{c} v_{C 1} .
\end{aligned}
$$

The membership functions used here are [18]

$$
\begin{aligned}
& M_{1}\left(v_{C 1}=1-\left(\frac{v_{C 1}}{d}\right)^{2}\right. \\
& M_{2}\left(v_{C 1}\right)=1-M_{1}\left(v_{C 1}\right)=\left(\frac{v_{C 1}}{d}\right)^{2} .
\end{aligned}
$$

The fuzzy rules for this model are

Rule 1: IF $v_{C 1}$ is $M_{1}\left(v_{C 1}\right)($ near 0$)$

$$
\text { THEN } \dot{\boldsymbol{x}}(t)=A_{1} \boldsymbol{x}(t)
$$

Rule 2: $\quad$ IF $v_{C 1}$ is $M_{2}\left(v_{C 1}\right)($ near $\pm d)$

$$
\text { THEN } \dot{\boldsymbol{x}}(t)=A_{2} \boldsymbol{x}(t)
$$

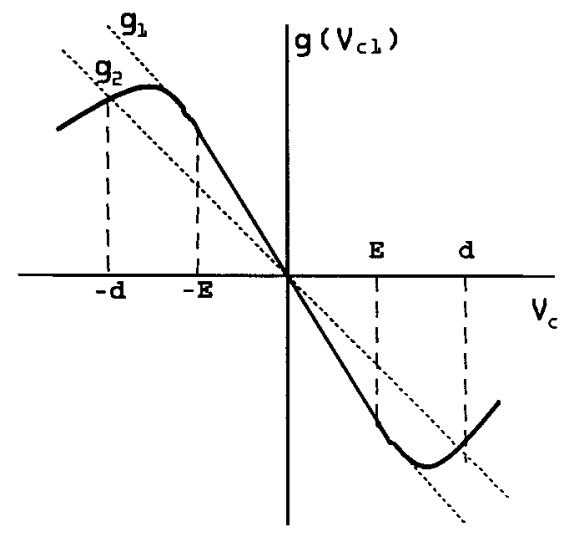

Fig. 4. Resistor characteristic for Case 2.

where

$$
A_{1}=\left[\begin{array}{ccc}
-\frac{1}{C_{1} R}-\frac{a}{C_{1}} & \frac{1}{C_{1} R} & 0 \\
\frac{1}{C_{2} R} & -\frac{1}{C_{2} R} & \frac{1}{C_{2}} \\
0 & -\frac{1}{L} & -\frac{R_{0}}{L}
\end{array}\right]
$$

and

$$
A_{2}=\left[\begin{array}{ccc}
-\frac{1}{C_{1} R}-\frac{m_{c}}{C_{1}} & \frac{1}{C_{1} R} & 0 \\
\frac{1}{C_{2} R} & -\frac{1}{C_{2} R} & \frac{1}{C_{2}} \\
0 & -\frac{1}{L} & -\frac{R_{0}}{L}
\end{array}\right] .
$$

III. OPTIMAL CONTROL AND INTELLIGENT

DIGITAL REDESIGN FOR HYBRID SYSTEMS

In this section, we formulate an optimal regional pole assignment scheme for the design of multiple fuzzy models and develop an intelligent digital redesign method by state matching in a hybrid state-space setting. The process of converting a continuous-time controller to an equivalent discrete-time controller is called digital redesign. The digital redesign technique can also be regarded as digital simulation, where the digital equivalence of a continuous-time system has to be found [21]. In digital control of continuous-time systems, the continuous-time state-space equations first need to be converted to discrete-time state-space equations. The feedback controller is then found by optimal pole-placement method in the continuous-time system and the state-matching method is finally introduced to convert the obtained continuous-time controller into a digital controller with slow-rate sampling.

\section{A. Optimal Control with Pole Placement}

Consider a linear controllable continuous-time subsystem in the $i$ th subspace described by

$$
\begin{aligned}
\dot{\boldsymbol{x}}_{c}(t) & =A_{i} \boldsymbol{x}_{c}(t)+B_{i} \boldsymbol{u}_{c}(t) \\
\boldsymbol{y}(t) & =C_{i} \boldsymbol{x}_{c}(t) \quad(i=1,2, \cdots, q) .
\end{aligned}
$$

The cost function for this subsystem is chosen to be

$$
J_{i}=\int_{0}^{\infty}\left[\boldsymbol{x}_{c}^{T}(t) Q_{i} \boldsymbol{x}_{c}(t)+\boldsymbol{u}_{c}^{T}(t) R_{i} \boldsymbol{u}_{c}^{i}(t)\right] d t
$$




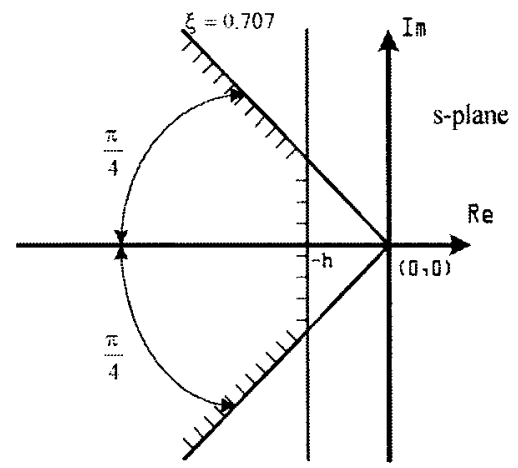

Fig. 5. Region of interest in the s-plane.

where $Q_{i}$ and $R_{i}$ are $n \times n$ nonnegative definite and $m \times m$ positive definite and symmetric matrices, respectively. The state-feedback control law $\boldsymbol{u}_{c}^{i}(t)$ in the $i$ th subspace (which minimizes the cost function) is expressed as

$$
\boldsymbol{u}_{c}^{i}(t)=-K_{c}^{i} \boldsymbol{x}_{c}(t)+E_{c}^{i} \boldsymbol{r}(t)=-R_{i}^{-1} B_{i}^{T} P_{i} \boldsymbol{x}_{c}(t)+E_{c}^{i} \boldsymbol{r}(t)
$$

where $K_{c}^{i}$ and $E_{c}^{i}$ are the feedback gain and the forward gain in the $i$ th subspace, respectively, $\boldsymbol{r}(t)$ is a reference input, and $P_{i}$ is a positive definite and symmetric matrix solution of the Riccati equation

$$
P_{i} B_{i} R_{i}^{-1} B_{i}^{T} P_{i}-P_{i} A_{i}-A_{i}^{T} P_{i}-Q_{i}=\mathbf{0}_{n}
$$

with $\left(Q_{i}, A\right)$ being detectable. Using this solution, the overall closed-loop system in the $i$ th subspace can be written as

$$
\dot{\boldsymbol{x}}_{c}(t)=\left(A_{i}-B_{i} K_{c}^{i}\right) \boldsymbol{x}_{c}(t)+B_{i} E_{c}^{i} \boldsymbol{u}_{c}^{i}(t)
$$

where $K_{c}^{i}=R_{i}^{-1} B_{i}^{T} P_{i}$ and the eigenvalues of $A_{i}-B_{i} K_{c}^{i}$ are located in the open left-half of the complex $s$-plane. Our objective is to determine $Q_{i}, R_{i}$, and $K_{c}^{i}$ such that the closedloop system (20) has its eigenvalues lying on or within the hatched region of Fig. 5.

Lemma 1 [14]: Let $\left(A_{i}, B_{i}\right)$ represent the given open-loop system in (16). Also let $h \geq 0$ represent the prescribed degree of relative stability as shown in Fig. 5. Then, the eigenvalues of the closed-loop system matrix $A_{i}-B_{i} R_{i}^{-1} B_{i}^{T} P_{i}$ lie to the left of the $-h$ vertical line, where the matrix $P_{i}$ is a solution of the Riccati equation

$$
\begin{aligned}
& P_{i} B_{i} R_{i}^{-1} B_{i}^{T} P_{i}-P_{i}\left(A_{i}+h \boldsymbol{I}_{n}\right)-\left(A_{i}+h \boldsymbol{I}_{n}\right)^{T} P_{i} \\
& =\mathbf{0}_{n} \quad(i=1,2, \cdots, q) .
\end{aligned}
$$

Note that the use of the degree of relative stability $h$ in (21) for finding the optimal control gain $\left(R_{i}^{-1} B_{i}^{T} P_{i}\right)$ is highly recommended since a subsystem of the TS fuzzy model is just an approximation of the original nonlinear system within the specific region of operation points.

Lemma 2 [14]: Suppose the given stable system matrix $A_{i} \in R^{n \times n}$ in (16) have eigenvalues $\hat{\lambda}_{j}^{-}\left(j=1, \cdots, \hat{n}^{-}\right)$ lying in the open hatched sector of Fig. 5 and eigenvalues $\hat{\lambda}_{j}^{+}\left(j=1, \cdots, \hat{n}^{+}\right)$outside that sector with $\hat{n}=\hat{n}^{-}+\hat{n}^{+}$. Consider the following two Riccati equations with assigned
$R_{i}>0$ :

$$
\begin{aligned}
\hat{Q}_{i} B_{i} R_{i}^{-1} B_{i}^{T} \hat{Q}_{i}-\hat{Q}_{i}\left(-A_{i}^{2}\right)-\left(-A_{i}^{2}\right)^{T} \hat{Q}_{i} & =\mathbf{0} \\
P_{i} B_{i} R_{i}^{-1} B_{i}^{T} P_{i}-P_{i} A_{i}-A_{i}^{T} P_{i}-\hat{Q}_{i} & =\mathbf{0}_{\hat{n}} .
\end{aligned}
$$

Then, the optimal closed-loop system matrix in the $i$ th subspace

$$
A_{c}^{i}=A_{i}-B_{i} K_{c}^{i}=A_{i}-\gamma_{i} B_{i}\left(R_{i}^{-1} B_{i}^{T} P_{i}\right)
$$

will enclose the invariant eigenvalues $\hat{\lambda}_{j}^{-}\left(j=1, \cdots \hat{n}^{-}\right)$and there exists at least one additional pair of complex conjugate eigenvalues lying inside the open sector of Fig. 5 if the constant gain $\gamma_{i}$ in (24) satisfies

$$
\gamma_{i} \geq \max \left\{\frac{1}{2}, \frac{b_{i}+\sqrt{b_{i}^{2}+a_{i} c_{i}}}{a_{i}}\right\}
$$

where $a_{i}=\operatorname{tr}\left[\left(B_{i} R_{i}^{-1} B_{i}^{T} P_{i}\right)^{2}\right], \quad b_{i}=\operatorname{tr}\left[B_{i} R_{i}^{-1} B_{i}^{T} P_{i} A_{i}\right]$, $c_{i}=\operatorname{tr}\left[B_{i} R_{i}^{-1} B_{i}^{T} \hat{Q}_{i}\right] / 2$, and $\operatorname{tr}[\cdot]$ denotes the trace of a matrix. If $c_{i}=0$, then all eigenvalues of $A_{c}^{i}$ lying in the $i$ th subspace have been optimally placed in the desired open sector of Fig. 5.

Since the poles of the system in the $i$ th subspace lie within the hatched sector region, the designed system has a prescribed relative stability in the $i$ th subspace. Although these local systems are individually stable, the global system may not be stable. This is because a controller in the $i$ th subspace is designed based on local performance only. We will further discuss and solve this problem in Section IV.

\section{B. Continuous-Time Local Controller Design Procedure}

The continuous-time design procedure for a local controller is described as follows.

Step 1: Set $i:=1$ and let $\left(A_{i}, B_{i}\right)$ be the given system matrices as in (16). Specify a value of $h$ and a weighting matrix $R>0$. Set $j=0$ and denote $A_{j}=A_{i}$ and $\gamma_{j}=1$ for $j=0$. Solve (21) for $P_{i}$ (denoted by $P_{j}$ ) satisfying (17) to obtain the closed-loop system matrix $A_{j+1}=A_{j}-B_{i} K_{j}$, where $K_{j}=\gamma_{j} R^{-1} B_{i}^{T} P_{j}$.

Step 2: Set $j:=1$ and solve (22) with $A_{i}:=A_{j}$ to find $\hat{Q}_{i}$ (denoted by $\hat{Q}_{j}$ ). If $c=\operatorname{tr}\left[B_{i} R^{-1} B_{i}^{T} \hat{Q}_{j}\right] / 2=0$, go to Step 4 .

Step 3: Solve (23) with $A_{i}:=A_{j}$ and $\hat{Q}_{i}:=\hat{Q}_{j}$ to determine $P_{i}$ (denoted by $P_{j}$ ) and obtain the closed-loop system matrix $A_{j+1}=A_{j}-B_{i} K_{j}$, where $K_{j}=\gamma_{j} R^{-1} B_{i}^{T} P_{j}$ and $\gamma_{j}$ is determined from (25). Go to Step 2.

Step 4: The desired optimal control law for the $i$ th subspace is

$$
\boldsymbol{u}_{c}(t)=-K_{c}^{i} \boldsymbol{x}_{c}(t)+E_{c}^{i} \boldsymbol{r}(t)
$$

where $K_{c}^{i}=\Sigma_{l=0}^{j-1} K_{l}$ and $E_{c}^{i}$ are the desired state-feedback gain and forward gain vectors in the $i$ th subspace, respectively.

Step 5: Set $i:=i+1$ and go to Step 1 if $i<q$, where $q$ is the number of fuzzy rules.

For tracking a reference input the cost function in (17) is written as

$$
\begin{aligned}
J= & \int_{0}^{\infty}\left\{\left[C_{i} \boldsymbol{x}_{c}(t)-\boldsymbol{r}(t)\right]^{T} Q_{e}\left[C_{i} \boldsymbol{x}_{c}(t)-\boldsymbol{r}(t)\right]\right. \\
& \left.\left.+\boldsymbol{u}_{c}^{T}(t) R \boldsymbol{u}_{c}(t)\right]\right\} d t
\end{aligned}
$$


where the weighting matrix $Q_{e}$ can be chosen from the aforementioned steps as

$$
Q_{e}=2 h P_{0}+\sum_{l=1}^{j}\left[\hat{Q}_{l}+\left(\gamma_{l}-1\right) P_{i} B_{i} R^{-1} B_{i}^{T} P_{l}\right] \gamma_{l}
$$

The desired optimal tracking control law is [14]

$$
\boldsymbol{u}_{c}^{i}(t)=-K_{e}^{i} \boldsymbol{x}_{e}(t)+E_{e}^{i} \boldsymbol{r}(t)
$$

where $K_{e}^{i}=R^{-1} B_{i}^{T} P_{e}$, and $P_{e}$ is the solution of

$$
A_{i}^{T} P_{e}+P_{e} A_{i}-P_{e}^{i} B_{i} R^{-1} B_{i}^{T} P_{e}+C_{i}^{T} Q_{e} C_{i}=\mathbf{0}_{\hat{n}}
$$

with

$$
E_{e}^{i}=-R^{-1} B_{i}^{T}\left(A_{i}-B_{i} K_{e}^{i}\right)^{-T} C_{i}^{T} / Q_{e}
$$

Note that when $C_{i}=\boldsymbol{I}_{\hat{n}}$, the feedback gain $K_{c}^{i}$ in (26) is identical to the $K_{e}^{i}$ in (29) and the forward gain $E_{c}^{i}$ in (26) can be chosen as the $E_{e}^{i}$ in (29).

\section{Intelligent Digital Redesign by State Matching}

For implementation of the digital control law, the continuous-time control law under slow-rate sampling obtained above is converted into an equivalent digital one with same-rate sampling. The digital redesign method matches the closed-loop state $x_{c}(t)$ at $t=k_{s} T_{s}$ with the digitally controlled state $x_{d}(t)$ at $t=k_{s} T_{s}$.

Using the closed-loop bilinear transformation method with a slow-rate sampling time $T_{s}$, the control law is developed as follows.

The equivalent discrete-time control law and state equation in the $i$ th subspace are described by

$$
\begin{aligned}
\boldsymbol{u}_{d}\left(k_{s} T_{s}\right)= & -K_{d}^{i} \boldsymbol{x}_{d}\left(k_{s} T_{s}\right)+E_{d}^{i} \boldsymbol{r}\left(k_{s} T_{s}\right) \\
& \left(k_{s} T_{s} \leq t<k_{s} T_{s}+T_{s}\right) \\
\boldsymbol{x}_{d}\left(k_{s} T_{s}+T_{s}\right)= & F_{i} \boldsymbol{x}_{d}\left(k_{s} T_{s}\right)+G_{i} \boldsymbol{u}_{d}\left(k_{s} T_{s}\right) \\
\boldsymbol{y}\left(k_{s} T_{s}\right)= & C_{i} \boldsymbol{x}_{d}\left(k_{s} T_{s}\right)
\end{aligned}
$$

where $F_{i}=e^{A_{i} T_{s}}$ and $G_{i}=\left(F_{i}-I_{n}\right) A_{i}^{-1} B_{i}$, where $\left[\left(F_{i}-I_{n}\right) A_{i}^{-1}\right]$ is always well defined.

Therefore, the closed-loop discrete-time system with the sampling time $T_{s}$ is

$$
x_{d}\left(k_{s} T_{s}\right)=\left(F_{i}-G_{i} K_{d}^{i}\right) x_{d}\left(k_{s} T_{s}\right)+G_{i} E_{d}^{i} r\left(k_{s} T_{s}\right)
$$

where $F_{i}$ and $G_{i}$ are the equivalent discrete-time state matrices, and $K_{d}^{i}$ and $E_{d}^{i}$ are the equivalent discrete-time feedback gain and forward gain in the $i$ th subspace, respectively.

Applying the block-pulse function method [14] to approximate $\boldsymbol{u}_{c}(t)$ over the interval $\left[k_{s} T_{s},\left(k_{s}+1\right) T_{s}\right]$ results in

$$
\boldsymbol{u}_{c}(t) \approx \sum_{j=0}^{\infty} \frac{1}{2}\left(u_{c}\left(j T_{s}\right)+\boldsymbol{u}_{c}\left(j T_{s}+T_{s}\right)\right) \phi_{j}(t)
$$

where $\phi_{j}(t)$ is the block-pulse function defined as follows:

$$
\phi_{j}(t)= \begin{cases}1, & \text { for } j T_{s} \leq t<(j+1) T_{s} \\ 0, & \text { otherwise }\end{cases}
$$

Using this block-pulse function, we obtain

$$
\begin{aligned}
\boldsymbol{x}_{c}\left(k_{s} T_{s}+T_{s}\right)= & F_{i} \boldsymbol{x}_{c}\left(k_{s} T_{s}\right)+\frac{1}{2} G_{i}\left[\boldsymbol{u}_{c}\left(k_{s} T_{s}\right)\right. \\
& \left.+\boldsymbol{u}_{c}\left(k_{s} T_{s}+T_{s}\right)\right]
\end{aligned}
$$

where $F_{i}=e^{A_{i} T_{s}}$ and $G_{i}=\left(F_{i}-I_{n}\right) A_{i}^{-1} B_{i}$, where $\left[\left(F_{i}-I_{n}\right) A_{i}^{-1}\right]$ is always well defined. Assume $\boldsymbol{r}(t)=\boldsymbol{r}\left(k_{s} T_{s}\right)$ for $k_{s} T_{s} \leq t<\left(k_{s}+1\right) T_{s}$. Substituting the discretized control law in (26) into the open-loop system in (37) results in the following approximated closed-loop system in the $i$ th subspace:

$$
\begin{aligned}
\boldsymbol{x}_{c}\left(k_{s} T_{s}+T_{s}\right)= & \left(I_{n}+\frac{1}{2} G_{i} K_{c}^{i}\right)^{-1}\left(F_{i}-\frac{1}{2} G_{i} K_{c}^{i}\right) \boldsymbol{x}_{c}\left(k_{s} T_{s}\right) \\
& +\left(I_{n}+\frac{1}{2} G_{i} K_{c}^{i}\right)^{-1} G_{i} E_{c}^{i} \boldsymbol{r}\left(k_{s} T_{s}\right) .
\end{aligned}
$$

Setting $\boldsymbol{x}_{c}\left(k_{s} T_{s}+T_{s}\right)$ and $\boldsymbol{x}_{c}\left(k_{s} T_{s}\right)$ in (38) equal to the corresponding $\boldsymbol{x}_{d}\left(k_{s} T_{s}+T_{s}\right)$ and $\boldsymbol{x}_{d}\left(k_{s} T_{s}\right)$ in (34) gives

$$
F_{i}-G_{i} K_{d}^{i}=\left(I_{m}+\frac{1}{2} G_{i} K_{c}^{i}\right)^{-1}\left(F_{i}-\frac{1}{2} G_{i} K_{c}^{i}\right)
$$

and

$$
G_{i} E_{d}^{i}=\left(I_{n}+\frac{1}{2} G_{i} K_{c}^{i}\right)^{-1} G_{i} E_{c}^{i} .
$$

The desired digital gains $\left(K_{d}^{i}, E_{d}^{i}\right)$ in (32) can be solved from (39) and (40) as follows:

$$
K_{d}^{i}=\frac{1}{2}\left(I_{m}+\frac{1}{2} K_{c}^{i} G_{i}\right)^{-1} K_{c}^{i}\left(F_{i}+I_{n}\right)
$$

where $K_{d}^{i}$ is the desired digitally redesigned feedback gain vector in the $i$ th subspace. Similarly, we can determine the desired digitally redesigned forward gain $E_{d}^{i}$ in the $i$ th subspace as

$$
E_{d}^{i}=\left(I_{m}+\frac{1}{2} K_{c}^{i} G_{i}\right)^{-1} E_{c}^{i} .
$$

We can then find the overall control law with $t=k_{s} T_{s}$ by defuzzifying $K_{d}^{i}$ and $E_{d}^{i}$ found in the $i$ th subspace, as shown in (63).

\section{Hybrid State-Space FuZZY Model-BASEd CONTROL SCHEME}

In digital control of continuous-time systems, the continuous-time state-space equations need to be converted into discrete-time state-space equations. In conventional TS fuzzy model-based controllers, sampling intervals for fuzzy modeling and controller design are often assumed to be the same. However, for practical realization of TS fuzzy model-based controllers, it is required to utilize a dual-rate sampling scheme; that is, the TS fuzzy model of a nonlinear system is first obtained with a fast sampling period (off-line or on-line). Then, a fast-rate sampling model has to be converted to a slow-rate sampling model. In this section, a model conversion method and stability conditions for the designed controller are formulated and the developed hybrid state-space fuzzy model-based control method is applied to the chaotic Chua circuit as an example of application. 


\section{A. Model Conversion}

To implement the obtained digital control law in (32), we need to convert a fast-rate sampling model into a slow-rate sampling model. For accurate fuzzy modeling and control, an appropriately small sampling period $T$ is chosen. In the dual-rate sampling method, this small sampling period $T$ is used as a fast-rate sampling period. Since the development of the above fuzzy control technique requires a slightly longer computational time in comparatively slow digital applications, it is necessary to choose an appropriate and relatively long sampling period $T_{s}$. This long sampling period (or slow-rate sampling period) is $N$ times the fast-rate sampling period, where $N$ is an integer.

Let the integer $N$ be the ratio between the slow sampling period $T_{s}$ and the fast sampling period $T$. There are many ways [19], [20] to convert $\left(F_{f i}, G_{f i}, C_{i}\right)$ to $\left(A_{i}, B_{i}, C_{i}\right)$ such as the Taylor series expansion method, direct truncation method, and matrix continued-fraction method, where $F_{f i}$ and $G_{f i}$ are fast-rate discrete-time model in the $i$ th subspace to be defined later.

The commonly used matrix continued-fraction method [19] for converting $\left(F_{f i}, G_{f i}, C_{i}\right)$ to $\left(A_{i}, B_{i}, C_{i}\right)$ is as follows:

$$
A_{i}=\frac{1}{T} \ln \left(F_{f i}\right) \cong \frac{2}{T} H_{i}
$$

where $H_{i} \equiv\left(F_{f i}-I_{n}\right)\left(F_{f i}+I_{n}\right)^{-1}$ and $F_{f i}=e^{A_{i} T}$. The matrix $B_{i}$ in the $i$ th subspace can be found as

$$
B_{i}=A_{i}\left(F_{f i}-I_{n}\right)^{-1} G_{f i}
$$

where $G_{f i}=\left(F_{f i}-I_{n}\right) A_{i}^{-1} B_{i}$ in which $\left[\left(F_{f_{i}}-I_{n}\right) A_{i}^{-1}\right]$ is always well defined.

It is remarked that for a piecewise-constant input, the stability of the converted analog and digital systems are invariant via the bilinear transform (which maps the left-half complex plane into the unit disk, preserving the stability).

Now, a fast-rate sampling model has to be converted into a slow-rate sampling model. The conversion can be carried out as follows:

$$
F_{i}=e^{A_{i} T_{s}}=\left(e^{A_{i} T}\right)^{N}=f_{f i}^{N}
$$

and

$$
G_{i}=\left(F_{i}-I_{n}\right) A^{-1} B_{i}=\left(F_{i}-I_{n}\right)\left(F_{f i}-I_{n}\right)^{-1} G_{f i} .
$$

\section{B. Stability Analysis of the Designed Controller and Controlled System}

The controller design problem is first to select $K_{c}^{i}$ and $E_{c}^{i}$ in (29) such that the local stability of the designed control system is satisfied (see Section III-A). As mentioned before, although these local systems are individually stable, we still need to ensure the globally controlled system be stable.

A well-known stability condition for the fuzzy system (3) is given as follows:

Theorem 1 [18]: The equilibrium of fuzzy system (3) is asymptotically stable in the large if there exists a common positive definite matrix $P_{c}$ such that

$$
A_{i}^{T} P_{c}+P_{c} A_{i}<0, \quad i=1,2, \cdots, q .
$$

Theorem 2 [18]: The equilibrium of fuzzy control system (7) is asymptotically stable in the large if there exits a common positive definite matrix $P_{c}$ such that the following two inequalities are satisfied:

$$
\begin{array}{rr}
\left(A_{i}-B_{i} K_{c}^{i}\right)^{T} P_{c}+P_{c}\left(A_{i}-B_{i} K_{c}^{i}\right)<0, & i=1,2, \cdots, q \\
& (48) \\
W_{i j}^{T} P_{c}+P_{c} W_{i j}<0, & 1 \leq i<j \leq q
\end{array}
$$

where

$$
W_{i j}=\frac{\left\{A_{i}-B_{i} K_{c}^{j}\right\}+\left\{A_{j}-B_{j} K_{c}^{i}\right\}}{2} \quad i<j
$$

for all $i$ and $j$, except the pairs $(i, j)$ that satisfy $\mu_{i}(\boldsymbol{x}) \mu_{j}(\boldsymbol{x})=$ 0 .

The above-mentioned stability condition of PDC design has drawbacks that stability analysis is not conducted for the original underlying nonlinear system (1) but for the simplified TS fuzzy model (2) instead and that the tracking problem is not explicitly addressed (at least in its earlier approach).

In order to solve these problems, we modify the controller rule of PDC with the same premise in (2) as follows, which is called an EPDC:

Controller Rule $i: \quad$ IF $x_{1}(t)$ is $M_{1}^{i}$ and $\cdots$ and $x_{n}(t)$ is $M_{n}^{i}$

$$
\begin{gathered}
\text { THEN } \boldsymbol{u}(t)=-K_{c}^{i} \boldsymbol{x}(t)+E_{c}^{i} \boldsymbol{r}(t) \\
(i=1,2, \cdots, q)
\end{gathered}
$$

where $K_{c}^{i}$ and $E_{c}^{i}$ are feedback gain and feedforward gain in the $i$ th subspace, respectively, and $\boldsymbol{r}(t)$ is the reference input.

Equation (51) can be analytically represented by

$$
\begin{aligned}
\boldsymbol{u}(t) & =\frac{\sum_{i=1}^{q} \omega_{i}\left(-K_{c}^{i} \boldsymbol{x}(t)+E_{c}^{i} \boldsymbol{r}(t)\right)}{\sum_{i=1}^{q} \omega_{i}} \\
& =-\sum_{i=1}^{q} \mu_{i} K_{c}^{i} \boldsymbol{x}(t)+\sum_{i=1}^{q} \mu_{i} E_{c}^{i} \boldsymbol{r}(t) \\
& =-K(\mu) \boldsymbol{x}(t)+E(\mu) \boldsymbol{r}(t) .
\end{aligned}
$$

The overall closed-loop system is then obtained from the feedback interconnection of the nonlinear system (1) and the controller (52), resulting in the following equation:

$$
\dot{\boldsymbol{x}}=\boldsymbol{F}_{o}(\boldsymbol{x}(t))+\boldsymbol{G}_{o}(\boldsymbol{x}(t)) \boldsymbol{r}(t)
$$

where $\boldsymbol{F}_{o}(\boldsymbol{x}(t))=\boldsymbol{f}(\boldsymbol{x}(t))-\boldsymbol{g}(\boldsymbol{x}(t)) K(\mu) \boldsymbol{x}(t)$ and $\boldsymbol{G}_{o}(\boldsymbol{x}(t)$, $E(\mu))=\boldsymbol{g}(\boldsymbol{x}(t)) E(\mu)$.

The following theorem is our main stability result for the equilibrium state of both the regulating and reference-tracking problems of the overall designed controller: 


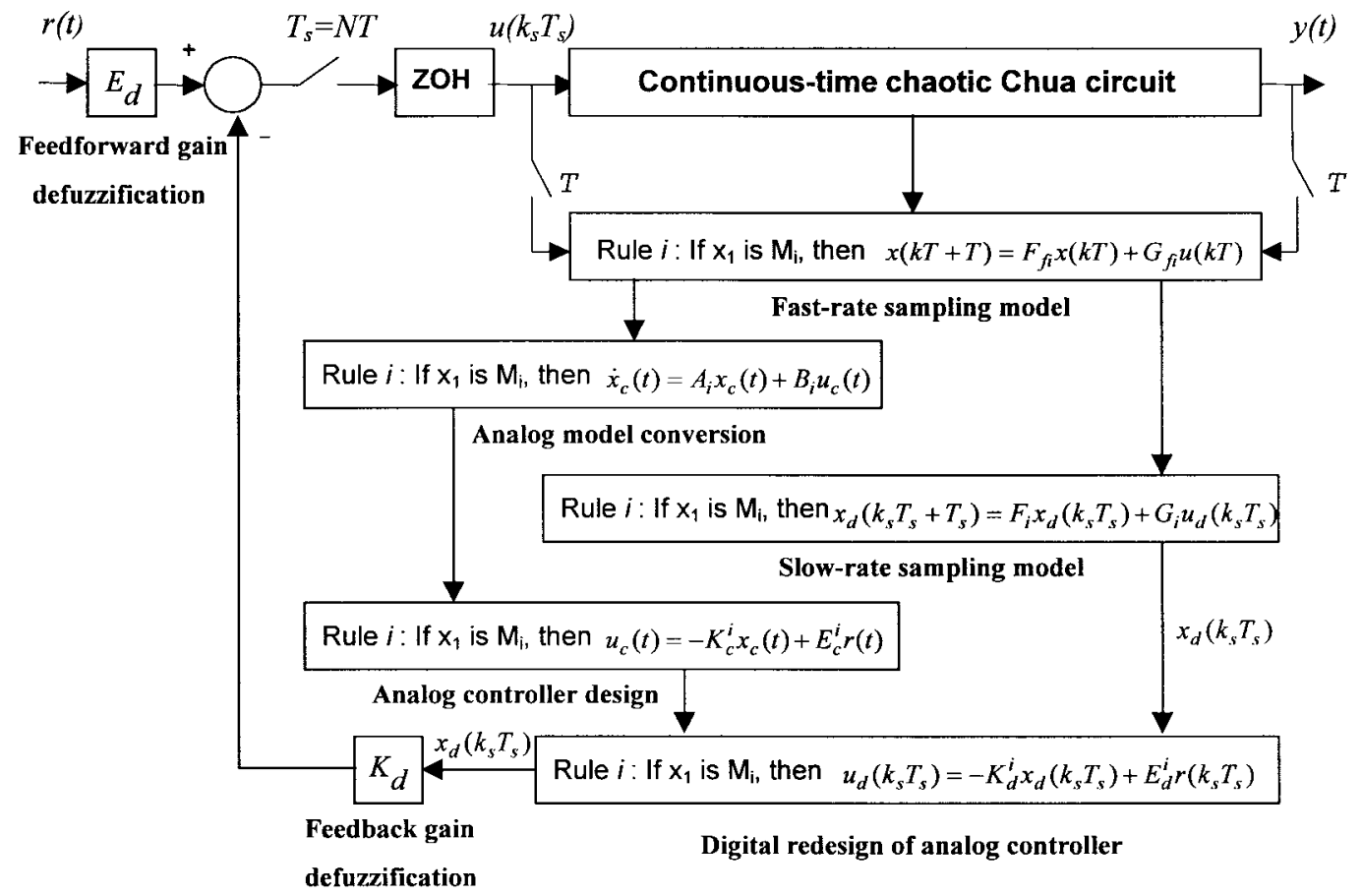

Fig. 6. Block diagram for intelligent digital redesign procedure.

Theorem 3: Consider the following nonlinear system:

$$
\dot{\boldsymbol{x}}(t)=A \boldsymbol{x}(t)-K(\mu) \boldsymbol{x}(t)+\boldsymbol{G}_{o}(\boldsymbol{x}(t), E(\mu)) \boldsymbol{r}(t)
$$

where $\boldsymbol{r}(t)$ is a given reference signal and $K(\mu)$ and $E(\mu)$ are control gain matrices with parameter $\mu \in \Re^{q}$, which can also be functions of $\boldsymbol{x}(t)$ and $t$ in general. If $K(\mu)$ and $E(\mu)$ are designed such that:

(i) the matrix $[A-K(\mu)]$ is stable uniformly for all $\boldsymbol{x} \in \Re^{n}$;

(ii) $\int_{0}^{\infty} \| \boldsymbol{G}_{o}(\boldsymbol{x}(t), E(\mu), \boldsymbol{r}(t) \| d t<\infty$; or

(iii) $\int_{0}^{\infty} \| \boldsymbol{G}_{o}(\boldsymbol{x}(t), E(\mu), \boldsymbol{r}(t)\|d t \leq\| C(\boldsymbol{x}(t), t)\|\| \boldsymbol{x}(t) \|$ with $\int_{0}^{\infty}\|C(\boldsymbol{x}(t), t)\| d t<\infty$

where $\|\cdot\|$ is the Euclidean norm, then the controlled system (54) is stable in the sense of Lyapunov.

Proof: See Appendix A.

Corollary 1: In the nonlinear control system (1) with a fuzzy controller (52), namely

$$
\dot{\boldsymbol{x}}(t)=\boldsymbol{F}_{o}(\boldsymbol{x}(t), K(\mu))+\boldsymbol{G}_{o}(\boldsymbol{x}(t), E(\mu)) \boldsymbol{r}(t)
$$

where

$$
\begin{aligned}
\boldsymbol{F}_{o}(\boldsymbol{x}(t), K(\mu)) & =\boldsymbol{f}(\boldsymbol{x}(t))-\boldsymbol{g}(\boldsymbol{x}(t)) K(\mu) \boldsymbol{x}(t) \\
G_{o}(\boldsymbol{x}(t), E(\mu)) & =\boldsymbol{g}(\boldsymbol{x}(t)) E(\mu)
\end{aligned}
$$

if the TS fuzzy model

$$
\dot{\boldsymbol{x}}(t)=[A-B K(\mu)] \boldsymbol{x}(t)
$$

is designed such that it can uniformly approximate the given uncontrolled system (55), namely $\| \boldsymbol{F}_{o}(\boldsymbol{x}(t), K(\mu))-[A-$ $B K(\mu)] \|$ can be arbitrary small [13] and if the control gains $K(\mu)$ and $E(\mu)$ are designed such that the two conditions (i) and (ii) [or (ii)' ${ }^{\prime}$ of Theorem 3 are satisfied, then the fuzzy control system (55) is stable in the sense of Lyapunov.
Proof: See Appendix B.

By Theorem 3 and Corollary 1, stability conditions of the tracking problem with reference input is as follows. For each fuzzy controller rule $\boldsymbol{r}(t)$ in the $i$ th subspace, a local controller is first designed based on consideration of local performance. Then, an linear matrix inequality (LMI) based stability analysis [23] is carried out to check whether the stability conditions (48) and (49) are satisfied and to find the common positive definite matrix $P_{c}$. Finally, we test whether the condition (ii) or (ii)' in Theorem 3 is satisfied.

The two controller gains $K$ and $E$ are designed to satisfy all these conditions. If the globally designed controller does not satisfy the stability conditions (i) and (ii) or (ii) ${ }^{\prime}$ in Theorem 3 , the controller $K_{c}^{i}$ and $E_{c}^{i}$ in (26) will be redesigned by changing the prescribed degree of relative stability $h$ as shown in Fig. 5. Note that the above stability is guaranteed not only for the TS fuzzy model (2), but also for the original underlying nonlinear system (1) due to Corollary 1.

\section{Hybrid State-Space Fuzzy Model-Based Control Procedure}

The above-described digital redesign methodology was first considered by Kuo [21] for a simple case. A digital statematching method was then proposed in [21] to solve the static digital redesign problem for linear systems and successfully applied to a simplified one-axis sky-lab satellite system. Shieh [4], [8], [9], [14] further improved the digital redesign method and investigated various types of (sub)optimal digital redesign methods. In this section, as an alternative of the approach suggested in [22], the digital redesign method and fuzzy inference system technique are combined for the control of complex such as chaotic systems. This new approach is called the intelligent digital redesign method.

Fig. 6 illustrates the entire intelligent digital redesign method, which uses the hybrid state-space fuzzy model-based 
control method to determine a control law. In this figure, $\mathrm{ZOH}$ is zero-order hold, $T_{s}$ is the slow-rate sampling period, $T$ is the fast-rate sampling period, and $q$ is the number of fuzzy rules used. The procedure of the continuous-time fuzzy modeling of Chua's circuit has been discussed in Section II. In order to find the overall control gains $K_{d}$ and $E_{d}$, the fuzzy inference rules for the control law are defuzzified by the center-average method.

Here, as an application example, we study digital control of the chaotic Chua circuit with control inputs produced by the digital redesign method developed in this paper.

Consider Chua's circuit with control inputs:

$$
\begin{aligned}
\dot{v}_{C 1} & =\frac{1}{C_{1}}\left(\frac{1}{R}\left(v_{C 2}-v_{C 1}\right)-g\left(g_{C 1}\right)\right)+u_{1} \\
\dot{v}_{C 2} & =\frac{1}{C_{2}}\left(\frac{1}{R}\left(v_{C 1}-v_{C 2}\right)+i_{L}\right)+u_{2} \\
\dot{i}_{L} & =\frac{1}{L}\left(-v_{C 1}-R_{0} i_{L}\right)+u_{3} .
\end{aligned}
$$

The design procedure for the overall hybrid state-space fuzzy model-based controller with dual-rate sampling as shown in Fig. 6 is described as follows.

Step 1: Choose appropriate dual-rate sampling periods $T$ and $T_{s}$ (=NT, $N$ is an integer) for fuzzy system modeling and model conversion for development of the control law in (18) and digital gains in (41) and (42). Specify a scalar $h$ and a positive definite and symmetric $m \times m$ matrix $R$ (Section IIIA). Given a pair $\{x(t), u(t)\}$ of the original continuous nonlinear system, find the fast-rate systems $\left\{F_{f i}, G_{f i}\right\}$ in (58) below for each fuzzy rule at time $t(k T \leq t<k T+T)$. In this example, the identified fuzzy model of the continuoustime Chua circuit is converted to a discrete-time state-space system with the fast-rate sampling period using (45) and (46). The resultant fast-rate sampling fuzzy model is as follows:

$$
\begin{array}{ll}
\text { Rule } i: & \text { IF } v_{C 1} \text { is } M_{1}\left(v_{C 1}\right) \\
& \text { THEN } x_{d}(k T+T)=F_{f i} x_{d}(k T)+G_{f i} \boldsymbol{u}_{d}(k T) \\
& (i=1, \cdots, q) .
\end{array}
$$

Step 2: Convert these fast-rate sampling fuzzy models $\left\{F_{f i}, G_{f i}\right\}$ into continuous-time models $\left\{A_{i}, B_{i}\right\}$ in (16) and discrete-time slow-rate models $\left\{F_{i}, G_{i}\right\}$ in (33) for each fuzzy rule, using (43)-(46) at time $t=k_{s} T_{s}$. In this step, we can use the fuzzy models identified in Section II. The resultant continuous-time state-space models and discrete-time state-space model are as follows.

\section{Continuous-Time State-Space Models}

$$
\begin{array}{ll}
\text { Rule } i: & \text { IF } v_{C 1} \text { is } M_{1}\left(v_{C 1}\right) \\
\text { THEN } \dot{\boldsymbol{x}}_{c}(t)=A_{i} \boldsymbol{x}_{c}(t)+B_{i} \boldsymbol{u}_{c}(t) \\
(i=1, \cdots, q) .
\end{array}
$$

\section{Discrete-Time State-Space Models}

$$
\begin{aligned}
& \text { Rule } i: \quad \text { IF } v_{C 1} \text { is } M_{1}\left(v_{C 1}\right) \\
& \operatorname{THEN} \dot{\boldsymbol{x}}_{d}\left(k_{s} T_{s}+T_{s}\right)=F_{i} \boldsymbol{x}_{d}\left(k_{s} T_{s}\right)+G_{i} \boldsymbol{u}_{d}\left(k_{s} T_{s}\right) \\
& (i=1, \cdots, q) .
\end{aligned}
$$

Step 3: Find the continuous-time state-feedback gain $K_{c}^{i}$ and forward gain $E_{c}^{i}$ in (18) for each fuzzy rule using continuous-time design procedure at time $t=k_{s} T_{s}$ (Section III-B). Stability analysis is then carried out to check whether the stability conditions defined by Theorem 3 are satisfied. In the case that some stability conditions are not satisfied, the controller $K_{c}^{i}$ and $E_{c}^{i}$ in (18) can be redesigned by incrementing $h$ by $\Delta h$, i.e., $h:=h+\Delta h$. The resultant continuous-time controller rules under the slow-rate sampling are as follows:

$$
\begin{aligned}
& \text { Rule } i: \quad \text { IF } v_{C 1} \text { is } M_{1}\left(v_{C 1}\right) \\
& \text { THEN } \boldsymbol{u}_{c}(t)=-K_{c}^{i} \boldsymbol{x}_{c}(t)+E_{c}^{i} \boldsymbol{r}_{c}(t), \\
& (i=1, \cdots, q) .
\end{aligned}
$$

Step 4: Find the digital constant state-feedback gain matrix $K_{d}^{i}$ and forward gain matrix $E_{d}^{i}$ in (32), using (41) and (42), from the available continuous-time state-feedback gains $K_{c}^{i}$ and forward gains $E_{c}^{i}$ in (18) for each fuzzy rule at time $t=k_{s} T_{s}$. The resultant discrete-time controller rules with the slow-rate sampling are as follows:

$$
\begin{array}{ll}
\text { Rule } i: & \text { IF } v_{C 1} \text { is } M_{1}\left(v_{C 1}\right) \\
& \text { THEN } \boldsymbol{u}_{d}\left(k_{s} T_{s}\right)=-K_{d}^{i} \boldsymbol{x}_{d}\left(k_{s} T_{s}\right)+E_{d}^{i} \boldsymbol{r}_{d}\left(k_{s} T_{s}\right) \\
& (i=1, \cdots, q) .
\end{array}
$$

The center-average method is then used as the defuzzifier to find the overall feedback gain $K_{d}$ and feedforward gain $E_{d}$ at time $t=k_{s} T_{s}$ as follows:

$$
K_{d}=\frac{\sum_{i=1}^{q} \omega_{i} K_{d}^{i}}{\sum_{i=1}^{q} \omega_{i}}, \quad E_{d}=\sum_{i=1}^{q} \omega_{i}
$$

where $w_{i}$ is the weight of the fuzzy set and $q$ is the number of the fuzzy rules.

Step 5: Find the digital control laws $\boldsymbol{u}_{d}\left(k_{s} T_{s}\right)$ in (32) using the gains in (63) at time $k_{s} T_{s}$. Go to Step 1 and repeat the process until control objective is achieved.

\section{Computer Simulations}

In this section, we present two simulation examples discussed in Section II-B to demonstrate the effectiveness of the proposed hybrid state-space fuzzy model-based control scheme for digital control of the chaotic Chua circuit. Simulations are performed using three control objectives: 1) to guide a chaotic or oscillatory trajectory to the origin; 2) to guide its trajectory to a constant reference; and 3) to guide its trajectory to a square wave reference, respectively. 


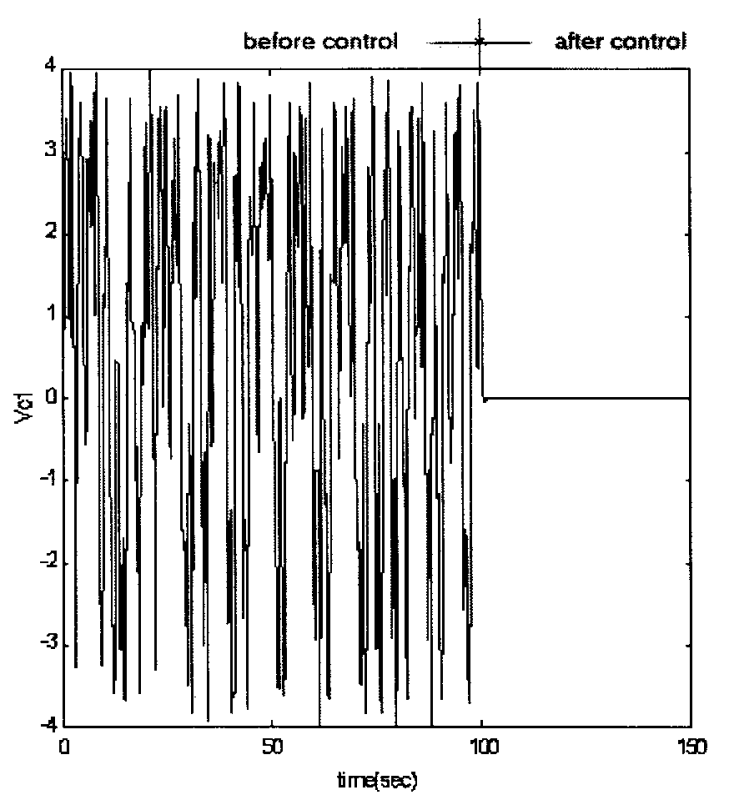

Fig. 7. Response of Chua's circuit with zero reference (Case 1).

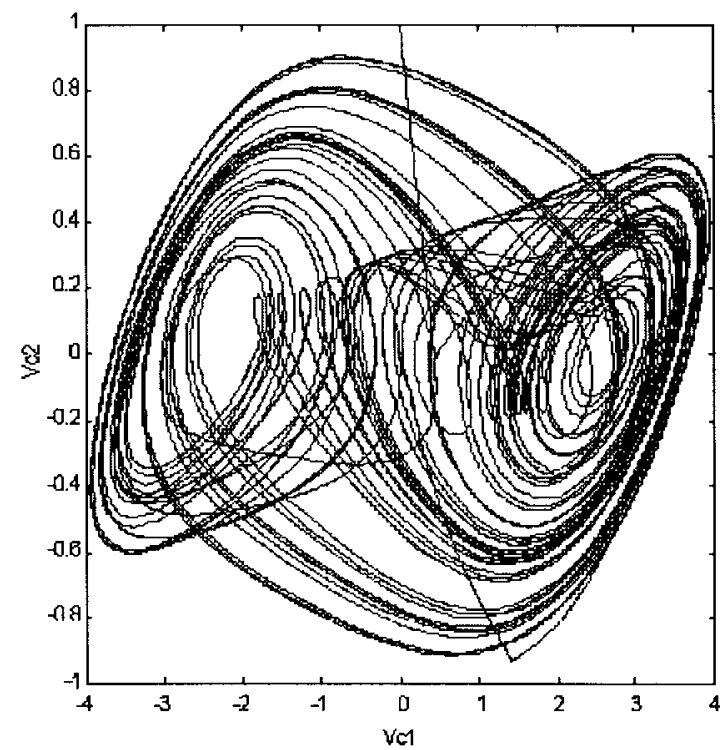

Fig. 8. Phase-plane trajectory of Chua's circuit with zero reference (Case 1).

Case 1: $g\left(v_{c 1}\right)$ is a piecewise-linear function.

Choose the chaotic parameters as follows:

$$
\begin{aligned}
R & =1, \quad R_{0}=0, \quad C_{1}=1 / 9, \quad C_{2}=1, \quad L=7 / 100 \\
m_{b} & =-5 / 7, \quad m_{a}=-8 / 7, \quad E=1, \quad d=5 .
\end{aligned}
$$

Let the fast-rate sampling period $T$ be $0.05 \mathrm{~s}$ and the slowrate sampling period $T_{s}(=N T: N=5)$ be $0.25 \mathrm{~s}$. The fuzzy model for the circuit is modeled with $T=0.05 \mathrm{~s}$ and the fuzzy controller is designed with $T_{s}=0.25 \mathrm{~s}$. The initial value of the prescribed degree of relative stability is $h=2.0$ and $\Delta h$ is 0.1 .

According to Section III-B, the feedback and feedforward gain matrices $K_{c}^{i}, E_{c}^{i}$ and the designed parameter $h$ are obtained as follows:

$$
\begin{aligned}
K_{c}^{1}= & {\left[\begin{array}{rrr}
7.7780 & 3.4972 & 2.9518 \\
3.4972 & 11.4150 & -0.8108 \\
2.9518 & -0.8108 & 2.3784
\end{array}\right] } \\
E_{c}^{1}= & {\left[\begin{array}{rrr}
6.4923 & -5.5028 & 2.9518 \\
2.4972 & 12.4150 & -1.8108 \\
2.9518 & 13.4749 & 2.3784
\end{array}\right] } \\
K_{c}^{2} & =\left[\begin{array}{rrr}
1.6536 & 0.3537 & 1.8628 \\
0.3537 & 10.7348 & -2.0889 \\
1.8628 & -2.0889 & 3.0116
\end{array}\right] \\
E_{c}^{2} & =\left[\begin{array}{rrr}
3.4536 & -8.6463 & 1.8628 \\
-0.6463 & 11.7348 & -3.0889 \\
1.8628 & 12.1968 & 3.0116
\end{array}\right] .
\end{aligned}
$$

In checking the stability of the fuzzy control system, we found the common positive definite matrix $P_{c}$ to be

$$
P_{c}=\left[\begin{array}{rrr}
0.2306 & 0.1106 & 0.0754 \\
0.1106 & 0.3085 & -0.1229 \\
0.0754 & -0.1229 & 0.2291
\end{array}\right] \text {. }
$$

The other conditions are also satisfied. Therefore, the overall continuous-time control system is stable in the sense of Lyapunov due to Corollary 1.

By the digital redesign formulas (41) and (42), the feedback and feedforward gain matrices $K_{d}^{i}$ and $E_{d}^{i}$ are obtained as follows:

$$
\begin{aligned}
K_{d}^{1} & =\left[\begin{array}{rrr}
3.8121 & 1.5114 & 1.4765 \\
1.1456 & 4.7682 & -0.0723 \\
1.5024 & -0.6709 & 1.2575
\end{array}\right], \\
E_{d}^{1} & =\left[\begin{array}{rrr}
2.5264 & -7.4886 & 1.4765 \\
0.1456 & 5.7682 & -1.0723 \\
1.5024 & 13.6148 & 1.2575
\end{array}\right] \\
K_{d}^{2} & =\left[\begin{array}{rrr}
0.9479 & -0.1812 & 1.1370 \\
0.5604 & 4.6477 & -0.2474 \\
0.9357 & -1.4311 & 1.5939
\end{array}\right] \\
E_{d}^{2} & =\left[\begin{array}{rrr}
2.7479 & -9.1812 & 1.1370 \\
-0.4396 & 5.6477 & -1.2474 \\
0.9357 & 12.8546 & -1.5939
\end{array}\right] .
\end{aligned}
$$

Therefore, the fuzzy rules for the control input are:

Rule 1: IF $v_{C 1}$ is $M_{1}\left(v_{C 1}\right)$

$$
\text { THEN } u_{d}\left(k_{s} T_{s}\right)=-K_{d}^{1} \boldsymbol{x}_{d}\left(k_{s} T_{s}\right)+E_{d}^{1} \boldsymbol{r}\left(k_{s} T_{s}\right)
$$

Rule 2: IF $v_{C 1}$ is $M_{2}\left(v_{C 1}\right)$

$$
\text { THEN } u_{d}\left(k_{s} T_{s}\right)=-K_{d}^{2} \boldsymbol{x}_{d}\left(k_{s} T_{s}\right)+E_{d}^{2} r\left(k_{s} T_{s}\right) .
$$

The overall feedback and feedforward gains $K_{d}$ and $E_{d}$ are calculated using (63).

Figs. 7-12 show the simulation results in this case. Fig. 7 shows the response of the circuit. The initial condition is $[0$ 


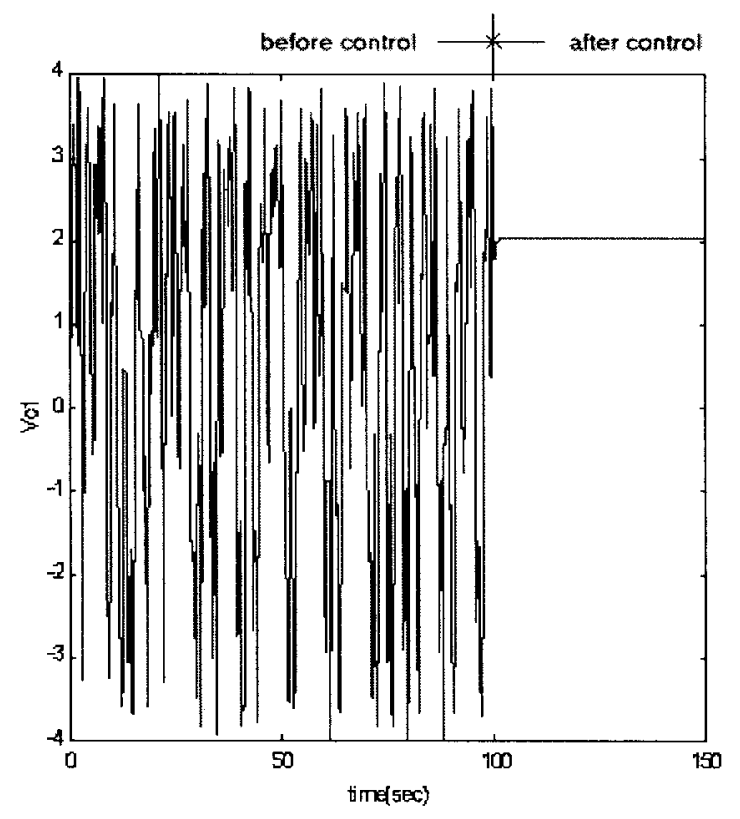

Fig. 9. Response of Chua's circuit with constant reference (Case 1).

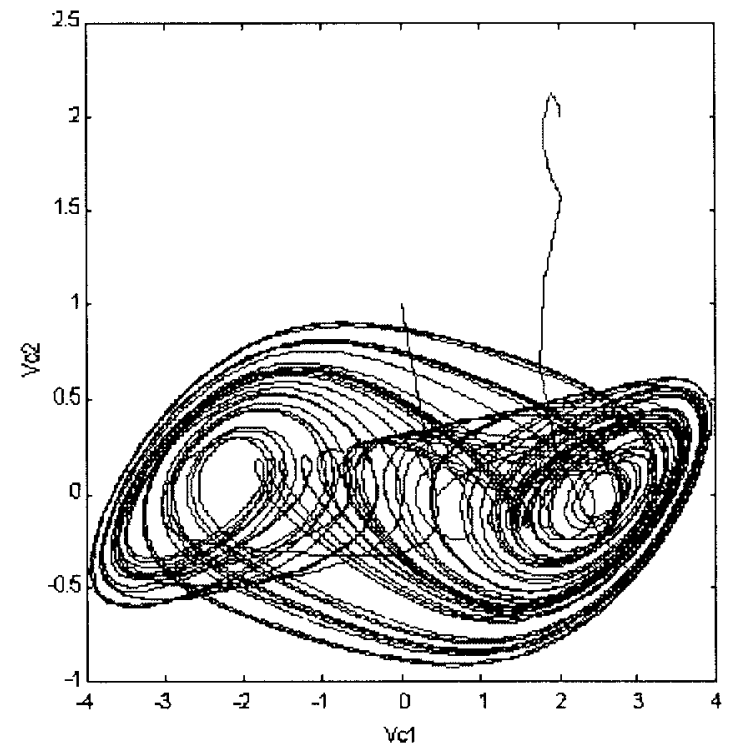

Fig. 10. Phase-plane trajectory of Chua's circuit with constant reference (Case 1).

$10]^{T}$ and the control is activated at $t=100$ for comparison purpose. Before the control mode is activated, the trajectory of the circuit is chaotic. However, after the controller is activated, the circuit trajectory is quickly directed to the origin. Fig. 8 shows the phase-plane trajectory of the same simulation result. The phase-plane trajectory starts with $(0,1)$ and $t=0$. Before $t=100$, it is clear that the trajectory does not approach the target in any way. After $t=100$, the trajectory goes to the origin.

The response of the Chua circuit with a constant reference $(r=2)$ is shown in Fig. 9. Before the control mode is activated, the trajectory is chaotic and highly oscillatory. But after the control mode is activated, the chaotic trajectory is quickly converged to the constant $(r=2)$. Fig. 10 shows the phase-plane trajectory for the same simulation. The phase-

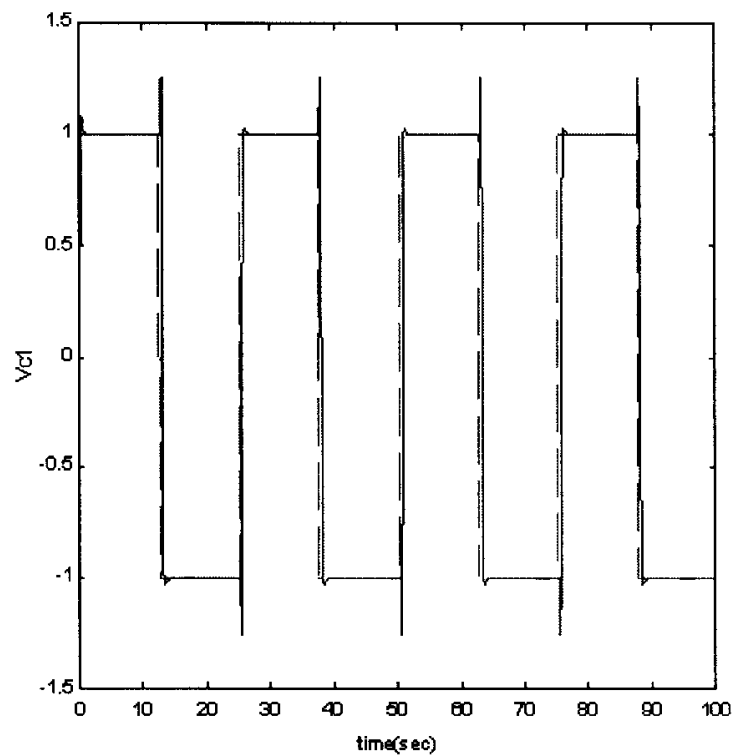

Fig. 11. Response of Chua's circuit with square wave reference (Case 1) dashed line: reference; solid line: output.

plane trajectory goes from initial point $(0,1)$ to reference point $(2,2)$.

Fig. 11 shows the result of the tracking control of following the square wave. According to the change of the reference input, the trajectory follows quite fastly. Fig. 12 shows the control input used for the square-wave tracking.

Case 2: $g\left(v_{c 1}\right)$ is a cubic function.

Choose the chaotic parameters as follows:

$$
\begin{aligned}
& R=10 / 7, \quad R_{0}=0, \quad C_{1}=1, \quad C_{2}=19 / 2 \\
& L=19 / 14, \quad a=-4 / 5, \quad c=2 / 45, \quad d=3 .
\end{aligned}
$$

Let the fast-rate sampling period $T$ be $0.05 \mathrm{~s}$ and the slowrate sampling period $T_{s}(=N T: N=5)$ be $0.25 \mathrm{~s}$. The fuzzy model for the circuit is modeled with $T=0.05 \mathrm{~s}$ and the fuzzy controller is designed with $T_{s}=0.25 \mathrm{~s}$. The initial value of the prescribed degree of relative stability is $h=2.0$ and $\Delta h$ is 0.1 .

Similar to Case 1, the feedback and feedforward gain matrices $K_{c}^{i}, E_{c}^{i}$, and the designed parameter $\mathrm{h}$ are obtained as follows:

$$
\begin{aligned}
K_{c}^{1} & =\left[\begin{array}{rrr}
4.0833 & 0.7562 & 0.1270 \\
0.7562 & 4.0993 & -0.6004 \\
0.1270 & -0.6004 & 3.8700
\end{array}\right] \\
E_{c}^{1} & =\left[\begin{array}{rrr}
3.9833 & 0.0862 & 0.1270 \\
0.6825 & 4.1730 & -0.7056 \\
0.1270 & 0.1365 & 3.8700
\end{array}\right] \\
K_{c}^{2} & =\left[\begin{array}{rrr}
3.2819 & 0.6875 & 0.1273 \\
0.6875 & 4.0998 & -0.5981 \\
0.1273 & -0.5981 & 3.8709
\end{array}\right] \\
E_{c}^{2} & =\left[\begin{array}{rrr}
3.5819 & -0.0125 & -0.1273 \\
0.6138 & 4.1735 & -0.7034 \\
0.1273 & 0.1387 & 3.89709
\end{array}\right], \quad h=1 .
\end{aligned}
$$




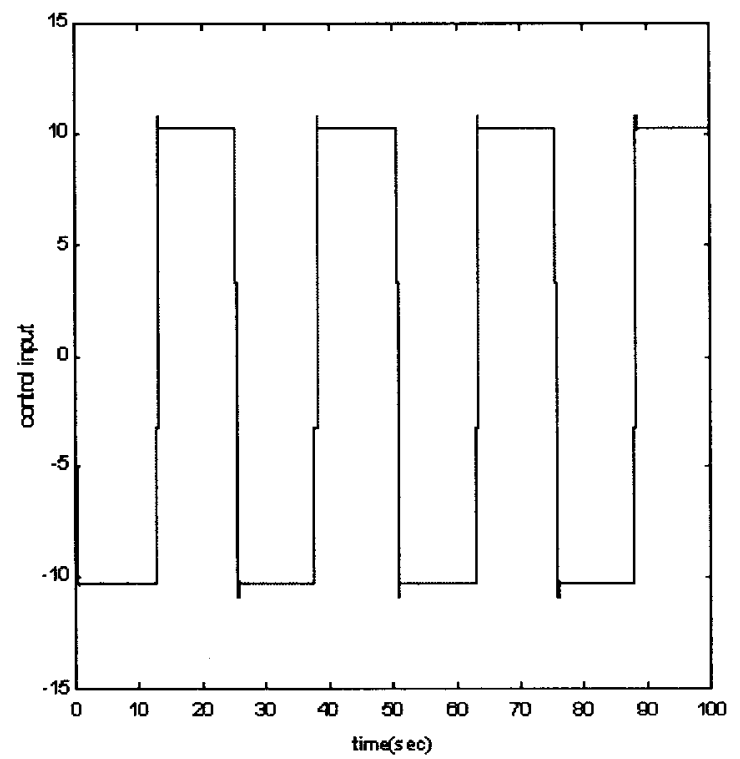

Fig. 12. Control signal of Chua's circuit for square wave tracking (Case 1).

A common positive definite matrix $P_{c}$ that satisfies stability condition defined by Theorem 3 is found to be

$$
P_{c}=\left[\begin{array}{rrr}
0.1992 & -0.0147 & -0.0091 \\
-0.0147 & 0.1689 & 0.0123 \\
-0.0091 & 0.0123 & 0.1853
\end{array}\right] \text {. }
$$

Therefore, the overall continuous-time control system is stable in the sense of Lyapunov due to Corollary 1.

The discrete-time feedback and feedforward gain matrices are obtained as follows:

$$
\begin{aligned}
K_{d}^{1} & =\left[\begin{array}{rrr}
2.6978 & 0.4837 & 0.0858 \\
0.3540 & 2.6951 & -0.2481 \\
0.0813 & -0.4236 & 2.5818
\end{array}\right] \\
E_{d}^{1} & =\left[\begin{array}{rrr}
2.5978 & -0.2163 & 0.0858 \\
0.2803 & 2.7688 & -0.3534 \\
0.0813 & 0.3132 & 2.5818
\end{array}\right] \\
K_{d}^{2} & =\left[\begin{array}{rrr}
2.2423 & 0.4520 & 0.0890 \\
0.3346 & 2.6971 & -0.2468 \\
0.0818 & -0.4222 & 2.5824
\end{array}\right] \\
E_{d}^{2} & =\left[\begin{array}{rrr}
2.5423 & -0.2480 & 0.0890 \\
0.2609 & 2.7708 & -0.3520 \\
0.0818 & 0.3147 & 2.5824
\end{array}\right] .
\end{aligned}
$$

The overall feedback and feedforward gains $K_{d}$ and $E_{d}$ are calculated by using (63).

Figs. 13-18 show the simulation results in this case. Fig. 13 shows the response of the circuit. The initial condition is $\left[\begin{array}{lll}0 & 1 & 0\end{array}\right]^{T}$ and the control is activated at $t=200$. Similarly to Case 1 , before the control mode $(t=200)$ is activated, the trajectory is chaotic and highly oscillatory. But after the control mode is activated, the trajectory is quickly guided to the origin. Fig. 14 illustrates the phase-plane trajectory of the circuit. The phase-plane trajectory starts with $(0,1)$ and $t=0$. Before $t=200$, it is clear that the trajectory does not approach

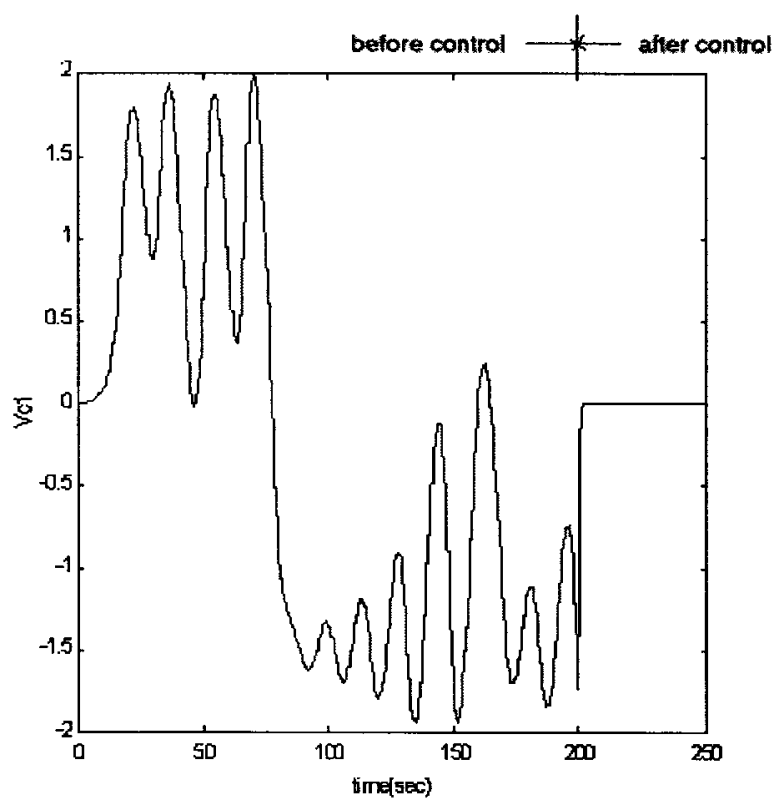

Fig. 13. Response of Chua's circuit with zero reference (Case 2).

the target in any way. After $t=200$, the trajectory goes to the origin.

The response of the circuit with constant reference $(r=2)$ is shown in Fig. 15. Before the control mode is activated, the trajectory is also chaotic and highly oscillatory. But after the control mode is activated, the trajectory is directed to the constant reference $(r=2)$. Fig. 16 shows the phase-plane trajectory for the same simulation.

The result of the tracking control to the square wave reference is presented in Fig. 17. According to the change of the reference input, the trajectory follows quite quickly. Fig. 18 shows the control signal for the square-wave tracking.

As seen in all these simulation results, the proposed digital control scheme is successful for digital control of the chaotic Chua circuit. Moreover, it is important to emphasize that under the given sufficient conditions, the stability is guaranteed not only for the TS fuzzy model but also for the original chaotic system.

\section{CONCLUSIONS}

In this paper, we have presented a hybrid state-space fuzzy model-based controller design methodology with dual-rate sampling for digital control of complex such as chaotic systems. TS fuzzy model is first used to model the dynamic system and then the extended parallel distributed compensation technique is proposed and formulated for the design of a fuzzy model-based controller. The optimal regional-pole assignment technique is also extended and adopted together with some new stability conditions to construct multiple local linear statespace models. In this design procedure, once the fuzzy model representation of the dynamic system is obtained we can apply the newly developed fuzzy control technique to design the digital controller for the dynamic system. It is emphasized that stability conditions are given not only for the TS fuzzy model but also for the underlying original dynamic system. This proposed technique presents a systematic and effective 


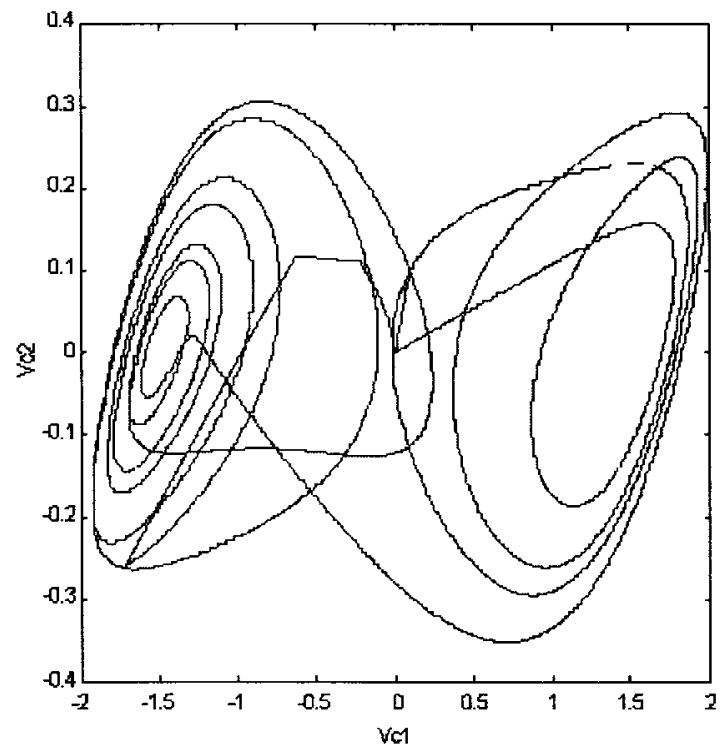

Fig. 14. Phase-plane trajectory of the Chua's circuit with zero reference (Case 2).

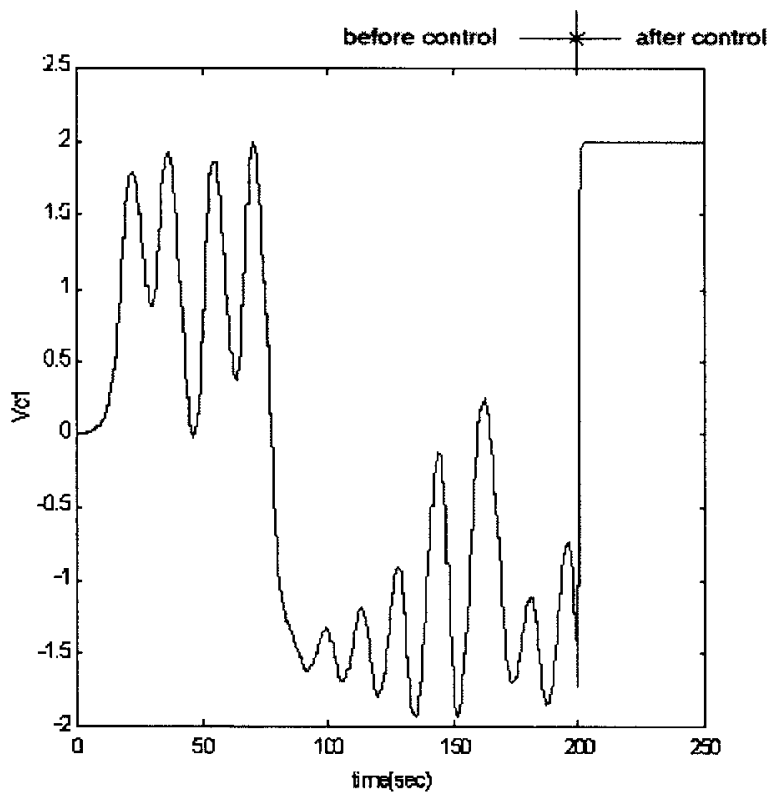

Fig. 15. Response of Chua's circuit with constant reference (Case 2).

framework for integration of modeling and digital control design for complex systems including chaotic systems. In order to demonstrate the effectiveness and feasibility of the proposed design method, we have simulated the chaotic Chua circuit that exhibits a wide variety of complicated dynamical phenomena such as bifurcations and chaos. Simulation results have shown that the new method can effectively control the chaotic circuit with a satisfactory performance. This hybrid state-space fuzzy model-based control scheme can also be applied to other complex nonlinear dynamical systems.

\section{APPENDIX A}

\section{PROOF OF THEOREM 3}

Let $\phi(t, 0)$ be the fundamental matrix of the linear system

$$
\dot{\boldsymbol{x}}(t)=[A-K(\mu)] \boldsymbol{x}(t) \text {. }
$$

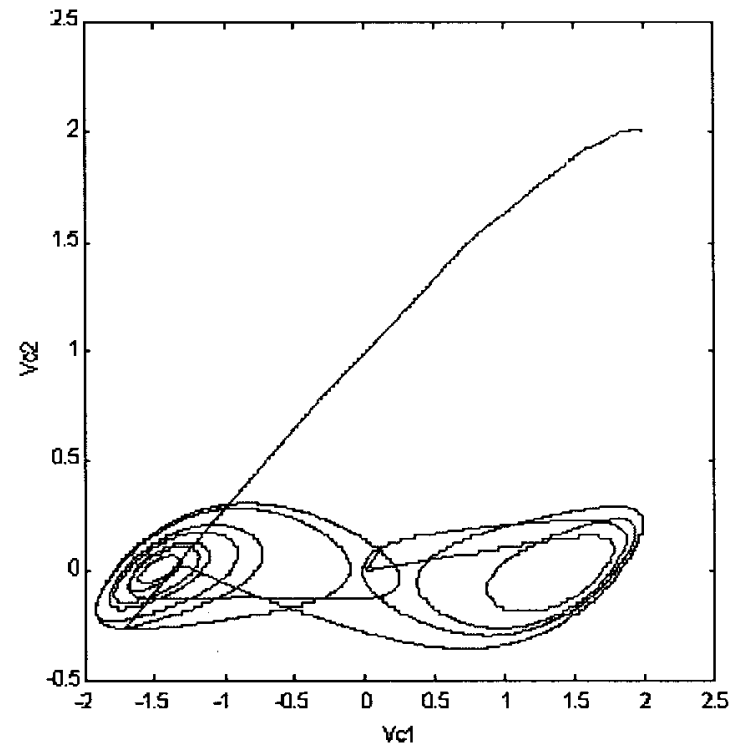

Fig. 16. Phase-plane trajectory of the Chua's circuit with constant reference (Case 2).

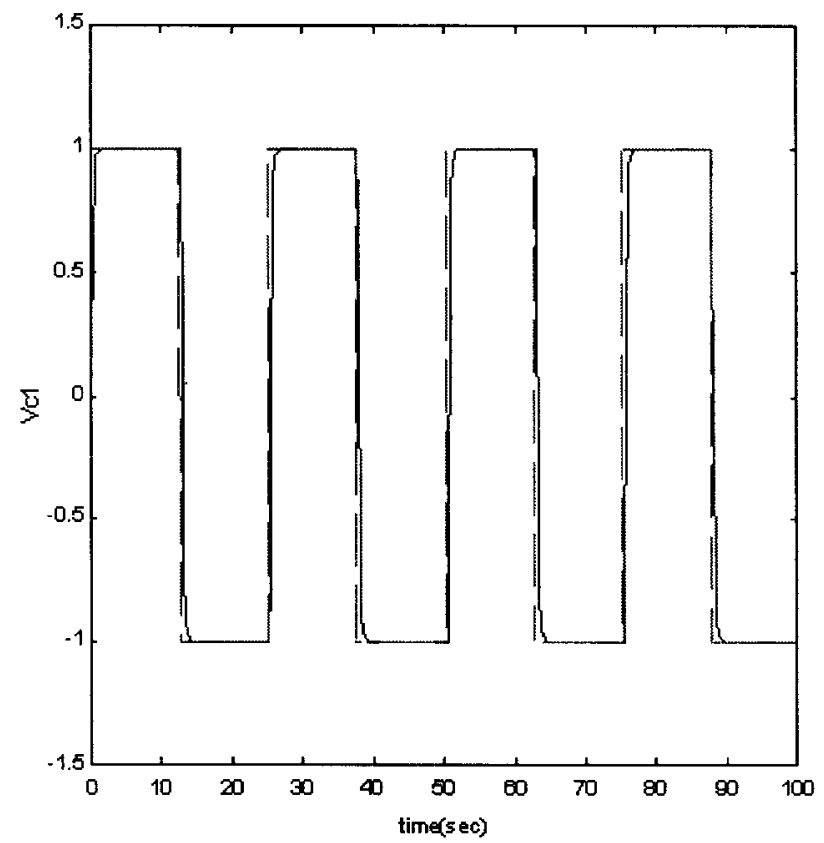

Fig. 17. Response of Chua's circuit with square-wave reference (Case 2) dashed line: reference; solid line: output.

Then, the general solution of system (54) is given by

$$
\boldsymbol{x}(t)=\phi(t, 0) \boldsymbol{x}_{0}+\int_{0}^{t} \phi(t, \tau) \boldsymbol{G}_{o}(\boldsymbol{x}(\tau), E(\mu)) \boldsymbol{r}(\tau) d \tau .
$$

Under condition (i) in Theorem 3, $\|\phi(t, \tau)\| \leq M<\infty$ for all $t, \tau \in[0, \infty)$, so that by condition (ii) in Theorem 3 , we have

$$
\begin{aligned}
\|\boldsymbol{x}(t)\| \leq & \|\phi(t, 0)\|\left\|\boldsymbol{x}_{0}\right\|+\int_{0}^{t}\|\phi(t, \tau)\| \\
& \cdot\left\|\boldsymbol{G}_{o}(\boldsymbol{x}(\tau), E(\mu)) \boldsymbol{r}(\tau)\right\| d \tau \\
\leq & M\left\|\boldsymbol{x}_{0}\right\|+\int_{0}^{\infty} M\|\boldsymbol{G}(\boldsymbol{x}(\tau), E(\mu)) \boldsymbol{r}(\tau)\| d \tau<\infty .
\end{aligned}
$$

Therefore, all solutions of system (54) are bounded. Under condition (ii)', the boundedness follows from the Gronwall 


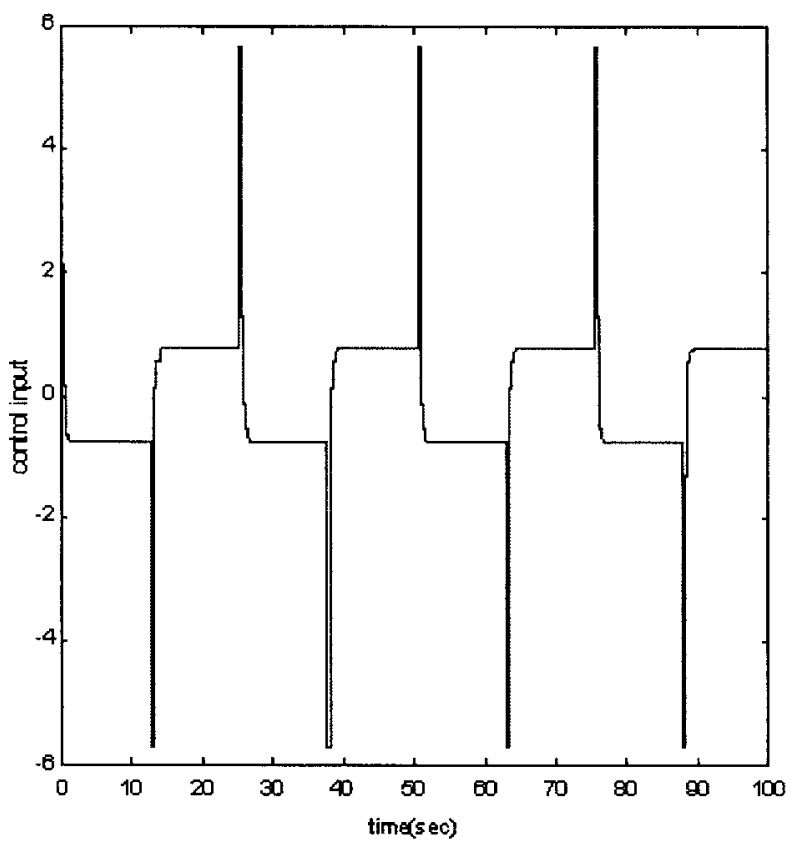

Fig. 18. Control signal of Chua's circuit for square-wave tracking (Case 2).

inequality [30] as follows:

$$
\begin{aligned}
\|\boldsymbol{x}(t)\| & \leq M\left\|x_{0}\right\|+\int_{0}^{t}\left\|G_{o}(\boldsymbol{x}(\tau), E(\mu)) \boldsymbol{r}(\tau)\right\| d \tau \\
& \leq M\left\|x_{0}\right\|+\exp \left\{\int_{0}^{\infty}\|C(\boldsymbol{x}(\tau), \tau)\| d \tau\right\}<\infty .
\end{aligned}
$$

Next, we can show that system (54) is stable in the sense of Lyapunov if and only if all the solutions of the system are bounded.

Indeed, if all solutions of the system are bounded, then there exists a constant $M$ such that $\|\phi(t, \tau)\| \leq M$ for all $t, \tau \in[0, \infty)$. Thus, for any $\epsilon>0,\left\|x_{0}-x_{1}\right\|<\epsilon / M$ implies

$\left\|\boldsymbol{x}\left(t ; \boldsymbol{x}_{0}\right)-\boldsymbol{x}\left(t ; \boldsymbol{x}_{1}\right)\right\|=\left\|\phi(t, 0)\left[x_{0}-x_{1}\right]\right\| \leq M\left\|x_{0}-x_{1}\right\|<\epsilon$

which means that the system is stable in the sense of Lyapunov.

Conversely, if the system is stable in the sense of Lyapunov, then for any $\epsilon>0$, there exists a $\delta>0$ such that $\left\|x_{1}\right\|<\delta$ implies

$$
\left\|x\left(t ; x_{1}\right)-0\right\|=\left\|\phi(t, 0) x_{1}\right\|<\epsilon .
$$

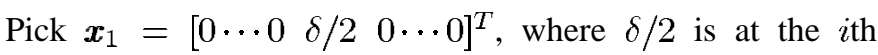
component. Then

$$
\left\|\phi(t, 0) \boldsymbol{x}_{1}\right\|=\left\|\phi_{i}(t, 0)\right\| \frac{\delta}{2}<\epsilon, \quad i=1, \cdots, n
$$

where $\phi_{i}(t, 0)$ is the $i$ th column of $\phi(t, 0)$. Therefore, $\left\|\phi_{i}(t, 0)\right\|<(2 n / \delta) \epsilon$, implying

$$
\left\|\boldsymbol{x}\left(t ; \boldsymbol{x}_{0}\right)\right\|=\left\|\phi(t, 0) \boldsymbol{x}_{0}\right\| \leq \frac{2 n}{\delta} \epsilon\left\|\boldsymbol{x}_{0}\right\|<\infty
$$

namely, all solutions of the system are bounded.

\section{APPENDIX B}

\section{PROOF OF COROLLARY 1}

It suffices to note that when the TS model (56) is used to uniformly approximate the given nonlinear fuzzy control system (55), we have

$$
\begin{aligned}
\dot{\boldsymbol{x}}(t)= & F_{o}(\boldsymbol{x}(t), K(\mu))-[A-B K(\mu)] \boldsymbol{x}(t) \\
& +[A-B K(\mu)] \boldsymbol{x}(t)+G_{o}(\boldsymbol{x}(t), E(\mu)) \boldsymbol{r}(t)
\end{aligned}
$$

so that by Theorem 3 , for any $\epsilon>0$, we have

$$
\begin{aligned}
\|\dot{\boldsymbol{x}}(t)\| \leq & \left\|F_{o}(\boldsymbol{x}(t), K(\mu))-[A-B K(\mu)]\right\| \\
& +\left\|[A-B K(\mu)]+G_{o}(\boldsymbol{x}(t), E(\mu)) \boldsymbol{r}(t)\right\| \\
\leq & \frac{\epsilon}{2}+\frac{\epsilon}{2}=\epsilon .
\end{aligned}
$$

\section{REFERENCES}

[1] G. Chen and X. Dong, From Chaos to Order-Methodologies, Perspectives, and Applications. Singapore: World Scientific, 1998.

[2] H. O. Wang, Tanaka, and M. F. Griffin, "Parallel distributed compensation of nonlinear systems by Takagi-Sugeno fuzzy model," in Proc. FUZZ-IEEE/IFES'95, Yokohama, Japan, Mar. 1995, pp. 531-538.

[3] P. J. Gathrop, "Hybrid self-tuning control," Proc. Inst. Elect. Eng., vol. 127 , pt. D, pp. 229-236, Sept. 1980.

[4] L. S. Shieh, X. M. Zhao, and J. Sunkel, "Hybrid state-space self-tuning control using dual-rate sampling," Proc. Inst. Elect. Eng., vol. 138, pt. D, pp. 50-58, Jan. 1991

[5] K. Warwick, "Self-tuning regulators-A state-space approach," Int. J. Contr. vol. 33, no. 5, pp. 839-858, 1981.

[6] H. Elliott, "Hybrid adaptive control of continuous time systems," IEEE Trans. Automat. Contr., vol. AC-27, pp. 419-426, Apr. 1982.

[7] K. Narendra, I. H. Kharlifa, and A. M. Annaswamy, "Error models for stable hybrid adaptive systems," IEEE Trans. Automat. Contr., vol. AC-30, pp. 339-347, Apr. 1985.

[8] L. S. Shieh, J. L. Zhang, and J. W. Sunkel, "A new approach to the digital redesign of continuous-time controllers," Contr. Theory Adv. Technol., vol. 8, pp. 37-57, 1992.

[9] L. S. Shieh, W. M. Wang, and M. K. Appu Panicker, "Design of PAM and PWM digital controllers for cascaded analog systems," ISA Trans., vol. 37, no, 3, pp. 201-213, 1998.

[10] J. S. Jang, "ANFIS: Adaptive-network-based fuzzy inference systems," IEEE Trans. Syst., Man, Cybern., vol. 23, pp. 665-684, Mar. 1993.

[11] T. Takagi and M. Sugeno, "Fuzzy identification of systems and its applications to modeling and control," IEEE Trans. Syst., Man, Cybern., vol. SMC-15, pp. 116-132, May 1985.

[12] K. Tanaka, T. Ikeda, and H. O. Wang, "A unified approach to controlling chaos via an LMI-based fuzzy control system design," IEEE Trans. Circuits Syst. $-I$, vol. 45, pp. 1021-1040, Oct. 1998

[13] L. X. Wang, "Fuzzy systems are universal approximators," in Proc. IEEE Int. Conf. Fuzzy Syst., San Diego, CA, Aug. 1992.

[14] L. S. Shieh, H. M. Dib, and S. Ganeson, "Continuous-time quadratic regulators and pseudo-continuous-time quadratic regulators with pole placement in a specific region," Proc. Inst. Elect. Eng., vol. 134, pt. D, no. 5, pp. 338-346, Sept. 1987.

[15] L. O. Chua, M. Komuro, and T. Matsumoto, "The double scroll family: I and II," IEEE Trans. Circuit Syst., vol. CS-33, pp. 1072-1118, 1986.

[16] S. H. Stogatz, Nonlinear Dynamics and Chaos. New York: AddisonWesley, 1994.

[17] G. Chen, "Intelligent identification and control of chaotic dynamics," in IEEE Symp. Circuits Syst., Atlanta, GA, May 1996, pp. 5-8.

[18] H. O. Wang, K. Tanaka, and T. Ikeda, "Fuzzy modeling and control of chaotic systems," in IEEE Symp. Circuits Syst., Atlanta, GA, May 1996, pp. 209-212.

[19] L. S. Shieh, J. S. H. Tsai, and S. R. Lian, "Determining continuous-time state equations from discrete-time state equations via the principle $q$ th root method," IEEE Trans. Automat. Contr., vol. AC-31, pp. 454-457, Mar. 1986.

[20] B. Kosko, Neural Networks for Signal Processing. Englewood Cliffs NJ: Prentice-Hall, 1991.

[21] B. C. Kuo, Digital Control Systems. New York: Holt, Rinehart, Winston, 1980

[22] J. Xu, G. Chen, and L. S. Shieh, "Digital redesign for controlling the chaotic Chua's circuit," IEEE Trans. Aerosp. Electron. Syst., vol. 32, pp. 1488-1499, Oct. 1996 
[23] H. O. Wang, K. Tanaka, and M. F. Griffin, "An approach to fuzzy control of nonlinear systems: Stability and design issues," IEEE Trans. Fuzzy Syst., vol. 4, pp. 14-23, Feb. 1996.

[24] K. Tanaka and M. Sugeno, "Stability analysis and design of fuzzy control systems," Fuzzy Sets Syst., vol. 45, no. 2, pp. 135-156, 1992.

[25] K. Tanaka, T. Ikeda, and H. O. Wang, "An LMI approach to fuzzy controller designs based on relaxed stability conditions," Proc. FUZZIEEE, Dec. 1997, pp. 171-176.

[26] S. G. Cao, N. W. Rees, and G. Feng, "Quadratic stability analysis and design of continuous-time fuzzy control system," Int. J. Syst. Sci., vol. 27, pp. 193-203, 1996

[27] "Stability analysis and design for a class of continuous-time fuzzy control systems," Int. J. Contr., vol. 64, pp. 1069-1087, 1996.

[28] _ "Analysis and design for a class of complex control systems-Parts I, II," Automatica, vol. 33, pp. 1017-1039, 1998.

[29] M. Sugeno and G. T. Kang, "Structure identification of fuzzy model," Fuzzy Sets Syst., vol. 28, pp. 15-33, 1988.

[30] R. Grimshaw, Nonlinear Ordinary Differential Equations. Boca Raton, FL: CRC, 1993.

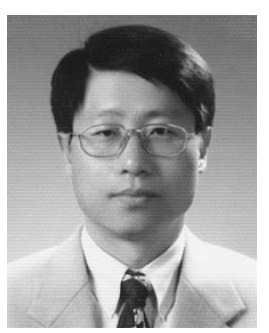

Young Hoon Joo was born in Sockcho, Korea, in 1958. He received the B.S., M.S., and Ph.D. degrees in electrical engineering from the Yonsei University, Korea, in 1982, 1984, and 1995, respectively.

He worked with Samsung Electronics Company, Korea, from 1986 to 1995 , as a Project Manager. He was with University of Houston, TX, from 1998 to 1999 , as a Visiting Professor in the Department of Electrical and Computer Engineering. He is currently Assistant Professor in the Department of Control and Instrumentation Engineering, the Kunsan National University, Korea, where he has been since 1995 . He has coauthored two research monographs and textbooks entitled Artificial Intelligence and Its Applications (Seoul, Korea: Bando, 1998, in Korean) and Introduction to AC/DC Circuits (Seoul, Korea: Dongmyung, 1999, in Korean). $\mathrm{He}$ also coauthored a chapter in The Handbook of Applied Computational Intelligence (Boca Raron, FL: CRC, to be published). In addition, he has published more than 80 journals and conference papers, mainly in the field of mobile robots, fuzzy modeling and control, genetic algorithms, intelligent control, and nonlinear systems control.

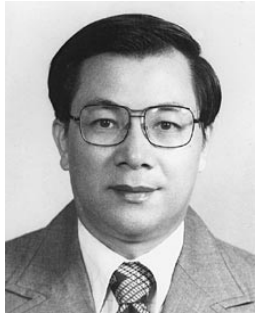

Leang-San Shieh (S'68-M'71-SM'92) received the B.S. degree from the National Taiwan University, Taiwan, in 1958, and the M.S. and $\mathrm{Ph} . \mathrm{D}$. degrees from the University of Houston, TX, in 1968 and 1970, respectively, all in electrical engineering.

He joined the Department of Electrical and Computer Engineering at the University of Houston in 1971 as an Assistant Professor, was promoted to Associate Professor in 1974, and Professor in 1978. Since 1988, he has been the Director of the Computer and System Engineering Program. He has directed 58 Masters' theses and 14 Ph.D. dissertations and has published more than 200 articles in various scientific journals. He coauthored the research monograph, An Algebraic Approach to Structural Analysis and Design of Multivariable Control Systems (New York: Springer-Verlag, 1988). His fields of interest are digital control, optimal control, self-tuning control, and hybrid control of uncertain systems.

Dr. Shieh was the recipient of more than ten College Outstanding Teacher Awards, the 1973 and 1997 College Teaching Excellence Awards, and the 1988 College Senior Faculty Research Excellence Award from the Cullen College of Engineering, University of Houston, and the 1976 University Teaching Excellence Award from the University of Houston. He also received the Honor of Merit from Instituto Universitario Politecnico, Republic of Venezuela, in 1978. He is a member of AIAA and is a Registered Professional Engineer in the state of Texas.

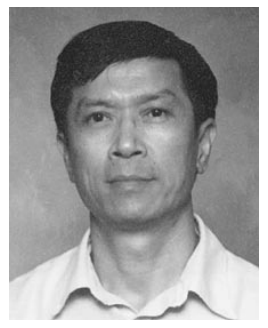

Guanrong (Ron) Chen (M'89-SM'92-F'97) received the M.Sc. degree in computer science from the Sun Yatsen (Zhongshan) University, China, and the Ph.D. degree in applied mathematics from Texas A\&M University, College Station, in 1981 and 1987, respectively.

His main research pursuit is in nonlinear systems-both dynamics and control. He is the coauthor of about 100 journal papers, numerous conference abstracts, and several research monographs and advanced textbooks, published since 1981 . He has served as Associate Editor for the Chinese Academy of Science Journal in Control Theory and Applications (since 1995) and as a Guest Editor for the International Journal of Bifurcation and Chaos, and the Journal of Information Science in the past. Among his publications are Kalman Filtering with Real-Time Applications (New York: Springer-Verlag, 1987, 1st ed.; 1998, 3rd ed.), Discrete H-Infinity Optimization (New York: SpringerVerlag, 1992, 1st ed.; 1997, 2nd ed.), Nonlinear Feedback Control Systems: An Operator Theory Approach (New York: Academic, 1993), Linear Stochastic Control Systems (Boca Raton, FL: CRC, 1995), Hopf Bifurcation Analysis: A Frequency Domain Approach (Singapore: World Scientific, 1996), and From Chaos to Order: Methodologies, Perspectives and Applications (Singapore: World Scientific, 1998).

Dr. Chen has served as an Associate Editor for the IEEE TRANSACTIONS ON Circuits AND Systems (1993-1995; 1999-2001). 